\title{
Broken Adaptive Ridge Regression for Right-Censored Survival Data
}

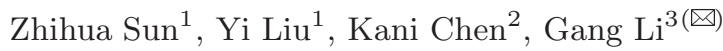 \\ ${ }^{1}$ Ocean University of China, ${ }^{2}$ Hong Kong University of Science and Technology and \\ ${ }^{3}$ University of California at Los Angeles
}

\begin{abstract}
Broken adaptive ridge (BAR) is a computationally scalable surrogate to $L_{0}$-penalized regression, which involves iteratively performing reweighted $L_{2}$ penalized regressions and enjoys some appealing properties of both $L_{0}$ and $L_{2}$ penalized regressions while avoiding some of their limitations. In this paper, we extend the BAR method to the semi-parametric accelerated failure time (AFT) model for right-censored survival data. Specifically, we propose a censored BAR (CBAR) estimator by applying the BAR algorithm to the Leurgan's synthetic data and show that the resulting CBAR estimator is consistent for variable selection, possesses an oracle property for parameter estimation and enjoys a grouping property for highly correlation covariates. Both low and high dimensional covariates are considered. The effectiveness of our method is demonstrated and compared with some popular penalization methods using simulations. Real data illustrations are provided on a diffuse large-B-cell lymphoma data and a glioblastoma multiforme data.
\end{abstract}

Keywords Accelerated failure time model · Grouping effect · $L_{0}$ penalization · Right censoring . Variable selection

\section{Introduction}

$L_{0}$-penalized regression, which directly penalizes the cardinality of a model, has been commonly used for variable selection in the low dimensional setting via well-known information criteria such as Mallow's $C_{p}$ (Mallows 1973), Akaike's information criterion (AIC) (Akaike 1974), the Bayesian information criterion (BIC) (Schwarz 1978; Chen and Chen 2008), and risk inflation criteria (RIC) (Foster and George 1994). It has also been shown to possess some optimal properties for variable selection and parameter estimation (Shen et al. 2012; Lin et al. 2010). However, $L_{0}$-penalization is also known to have some limitations such as being computationally NP-hard, not scalable to high dimensional data, and unstable for variable selection (Breiman 1996). To overcome these shortcomings, the broken adaptive ridge (BAR) method (Dai et al. 2018a,b) has been recently introduced as a surrogate to $L_{0}$ penalization for simultaneous variable selection and parameter estimation under the linear model (Dai et al. 2018a,b). It was noted by Dai et al. (2018a,b) that the BAR estimator, defined as the limit of an iteratively reweighted $L_{2}$ (ridge) penalization algorithm, retains some appealing properties of $L_{0}$ penalization while avoiding its pitfalls. For instance, BAR generally yields a more sparse, accurate, and interpretable model than some popular $L_{1}$-type penalization methods such as LASSO and its various variations, while maintaining comparable prediction performance. Moreover, unlike the exact $L_{0}$ penalization, BAR is computationally scalable to high dimensional covariates and is stable for variable selection. Lastly, in addition to being consistent for variable selection and oracle for parameter estimation, the BAR estimator enjoys a grouping property for highly correlated covariates, a desirable feature not shared by most other oracle variable selection procedures.

Because of its appealing properties, the BAR penalization method has been recently extended to the Cox (1972) model with censored survival data (Kawaguchi et al. 2019; Zhao et al. 2019) via penalized likelihood. However, it is well known that the Cox (1972) proportional hazards assumption do not always hold in practice. Thus it is desirable to extend the BAR penalization method to other common survival regression models. This paper studies an extension of the BAR penalization method 
to the semi-parametric accelerated failure time (AFT) model, a popular alternative to the Cox model for right censored survival data. To this end, we note that the semi-parametric AFT model is a linear model for the log-transformed survival time with a completely unspecified error distribution, for which the likelihood approach does not yield a consistent parameter estimator even for the classical uncensored linear regression model. Hence, the BAR penalized likelihood methods of Kawaguchi et al. (2019) and Zhao et al. (2019) for the Cox (1972) do not apply to the semiparmetric AFT model. A different approach would be required.

In this paper, we propose an extension of the BAR penalization method to the semi-parametric AFT model by coupling the Leurgans (1987) synthetic data approach with the BAR penalty, study its large sample properties, and demonstrate it effectiveness in comparison with some popular penalization methods using simulations. Specifically, we first use the Leurgans (1987) synthetic variable method to construct a synthetic outcome variable and then apply the BAR method for uncensored linear regression (Dai et al. 2018b) to the synthetic outcome variable. We then give sufficient conditions under which the proposed censored BAR (CBAR) estimator is consistent for variable selection, behaves asymptotically as well as the oracle estimator based on the true reduced model, and possesses a grouping property for highly correlated covariates. We also combine BAR with a sure joint screening method to obtain a two-step variable selection and parameter estimation method for ultrahigh dimensional covariates. Not surprisingly, our simulations demonstrate that the proposed CBAR method generally yields a more sparse and more accurate model as compared to some other popular penalization methods such as LASSO, SCAD, MCP, and adaptive LASSO within the Leurgans (1987) synthetic data framework, which is consistent with the findings of Dai et al. (2018b) for uncensored data. Lastly, we have implemented the proposed CBAR method in an $\mathrm{R}$ package, named CenBAR, and made it publicly available at https://CRAN.R-project.org/package=CenBAR.

Before going further, we note that there exist a number of other variable selection methods in the literature for the semiparametric the AFT model with right censored data. These methods are derived by combining various penalization methods such as LASSO with different extensions of the least squares principle for right censored data. For example, the Lasso, bridge, elastic net or MCP penalties have been combined with the Stute (1993) weighted least squares method (Huang et al. 2006; Huang and Ma 2010; Datta et al. 2007); and the Dantzig, elastic net, Lasso, adaptive Lasso and SCAD penalties have been combined with the Buckley and James (1979) method (Yi and Li 2009; Wang et al. 2008; Johnson et al. 2008; Johnson 2009). This paper makes a unique theoretical contribution since neither the BAR penalization nor the Leurgans (1987) synthetic data method has been previously rigorously studied in the context of variable selection for the semiparametric the AFT model. We also illustrate and compare empirically the BAR penalization versus some popular penalization methods when the Leurgans (1987) synthetic data least squares method is used. We do not compare different penalization methods when they are coupled with different censored least squares methods because different censored least squares methods are derived under different conditions and none is expected to dominate another across all scenarios.

The rest of the paper is organized as follows. In Section 2, we define our CBAR estimator and state its theoretical properties. We also discuss how to handle ultra-high dimensional covariates. In Section 3, we evaluate the finite sample performance of CBAR in comparison with other penalization methods via extensive simulations. In Section 4, we illustrate the CBAR method on a diffuse largeB-cell lymphoma data and a glioblastoma multiforme data with high dimensional covariates. Proofs of the theoretical results are provided in the appendix. 


\section{Censored broken adaptive ridge (CBAR) regression}

2.1 Notations and preliminaries

\subsubsection{Model and data}

Consider the linear regression model

$$
Y_{i}=\mathbf{x}_{i}^{\top} \boldsymbol{\beta}+\varepsilon_{i}, \quad i=1,2, \ldots, n,
$$

where for the $i$ th subject, $Y_{i}$ denotes the response variable, $\mathbf{x}_{i}$ is the $p_{n}$-vector random covariates, $\boldsymbol{\beta}=\left(\beta_{1}, \ldots, \beta_{p_{n}}\right)^{\top}$ is a vector of regression coefficients, and $\varepsilon_{i}$ is i.i.d. error term with an unknown error distribution, $E\left(\varepsilon_{i}\right)=0$ and $\operatorname{Var}\left(\varepsilon_{i}\right)=\sigma^{2}<\infty$. Model (1) is commonly referred to as the accelerated failure time (AFT) model when $Y$ is the log-transformed survival time (Kalbfleisch and Prentice 2002).

Without loss of generality, assume that $\boldsymbol{\beta}_{0}=\left(\boldsymbol{\beta}_{01}^{\top}, \boldsymbol{\beta}_{02}^{\top}\right)^{\top}$ is the true value of $\boldsymbol{\beta}$, where $\boldsymbol{\beta}_{01}$ is a $q \times 1$ nonzero vector and $\boldsymbol{\beta}_{02}$ is a $\left(p_{n}-q\right) \times 1$ zero vector. We further assume the columns of the design matrix $\mathbf{X}=\left(\mathbf{x}_{1}, \ldots, \mathbf{x}_{n}\right)^{\top}$ have mean zero and unit $L_{2}$-norm. Throughout the paper, $\|\cdot\|$ represents the Euclidean norm for a vector and spectral norm for a matrix.

Assume that one observes a right censored data consisting of $n$ independent and identically distributed triples $\left(T_{i}, \delta_{i}, \mathbf{x}_{i}\right), i=1, \ldots, n$, where for the $i$ th subject, $T_{i}=\min \left(Y_{i}, C_{i}\right)$ is the observation time, $\delta_{i}=I\left(Y_{i} \leq C_{i}\right)$ is a censoring indicator, $C_{i}$ is the i.i.d. censoring time with the distribution function $H . C_{i}$ is assumed to be independent of $Y_{i}$ and $\mathbf{x}_{i}$.

\subsubsection{Broken adaptive ridge (BAR) for uncensored data}

For reader convenience, we first briefly review the broken adaptive ridge (BAR) estimator of Dai et al. (2018b) for simultaneous variable selection and parameter estimation with the uncensored data $\mathbf{Y}$ and $\mathbf{X}$, where $\mathbf{Y}=\left(Y_{1}, \ldots, Y_{n}\right)^{\top}$.

Following the notations of Dai et al. (2018b), the BAR estimator of $\boldsymbol{\beta}$ based on $\mathbf{Y}$ and $\mathbf{X}$ is a surrogate $L_{0}$-penalized estimator defined as the limit of the following iteratively reweighted ridge regression algorithm:

$$
\begin{aligned}
\boldsymbol{\beta}^{(k)} & =\arg \min _{\boldsymbol{\beta}}\left\{\|\mathbf{Y}-\mathbf{X} \boldsymbol{\beta}\|^{2}+\lambda_{n} \sum_{j=1}^{p_{n}} \frac{\beta_{j}^{2}}{\left\{\boldsymbol{\beta}_{j}^{(k-1)}\right\}^{2}}\right\} \\
& =\left\{\mathbf{X}^{\top} \mathbf{X}+\lambda_{n} \mathbf{D}\left(\boldsymbol{\beta}^{(k-1)}\right)\right\}^{-1} \mathbf{X}^{\top} \mathbf{Y}, \quad k=1,2, \ldots
\end{aligned}
$$

where $\boldsymbol{\beta}^{(0)}=\arg \min _{\boldsymbol{\beta}}\left\{\|\mathbf{Y}-\mathbf{X} \boldsymbol{\beta}\|^{2}+\xi_{n} \sum_{j=1}^{p_{n}} \beta_{j}^{2}\right\}=\left(\mathbf{X}^{\top} \mathbf{X}+\xi_{n} \mathbf{I}\right)^{-1} \mathbf{X}^{\top} \mathbf{Y}$ is an initial ridge estimator, $\xi_{n}>0$ and $\lambda_{n} \geq 0$ are tuning penalization parameters, and for any $p_{n}$-dimensional vector $\boldsymbol{\theta}=\left(\theta_{1}, \ldots, \theta_{p_{n}}\right)^{T}, \mathbf{D}(\boldsymbol{\theta})=\operatorname{diag}\left(\frac{1}{\theta_{1}^{2}}, \ldots, \frac{1}{\theta_{p_{n}}^{2}}\right)$. Note that each reweighted $L_{2}$ penalty can be regarded as an adaptive surrogate $L_{0}$ penalty and the approximation of $L_{0}$ penalization improves with each iteration. Dai et al. (2018b) showed that the BAR estimator $\hat{\boldsymbol{\beta}}=\lim _{k \rightarrow \infty} \boldsymbol{\beta}^{(k)}$ is selection consistent and possesses an oracle property: if the true model is sparse with some zero coefficients, then with probability tending to 1 , BAR estimates the true zero coefficients as zeros and estimates the non-zero coefficients as well as the scenario when the true sub-model is known in advance. 
2.2 Broken adaptive ridge estimator for censored data (CBAR)

For right censored data, the above BAR algorithm is obviously not applicable since one only observes $\left(T_{i}, \delta_{i}\right)$ instead of $Y_{i}$. To overcome the problem, we propose to adopt the Leurgans (1987) synthetic data approach for censored linear regression to variable selection by first transforming $\left(T_{i}, \delta_{i}\right)$ into a synthetic variable $Y_{i}^{*}$ and then applying the BAR method to the synthetic data variable $Y_{i}^{*}$. Specifically, the Leurgans (1987) synthetic data $Y_{i}^{*}$ is defined as

$$
Y_{i}^{*}=\int_{-\infty}^{T^{n}}\left(\frac{I\left(T_{i} \geq s\right)}{1-\hat{H}(s)}-I(s<0)\right) d s
$$

where $T^{n}=\max \left\{T_{1}, \ldots, T_{n}\right\}$ and $\hat{H}$ is the Kaplan-Meier estimator of $H$. To apply the BAR method to synthetic data $Y_{i}^{*}$, let $\mathbf{Y}^{*}=\left(Y_{1}^{*}, \ldots, Y_{n}^{*}\right)^{\top}$ and define an initial ridge estimator

$$
\hat{\boldsymbol{\beta}}^{(0)}=\left(\mathbf{X}^{\top} \mathbf{X}+\xi_{n} \mathbf{I}\right)^{-1} \mathbf{X}^{\top} \mathbf{Y}^{*}
$$

and then, for $k \geq 1$,

$$
\hat{\boldsymbol{\beta}}^{(k)}=g\left(\hat{\boldsymbol{\beta}}^{(k-1)}\right),
$$

where

$$
g(\tilde{\boldsymbol{\beta}})=\arg \min _{\boldsymbol{\beta}}\left\{\left\|\mathbf{Y}^{*}-\mathbf{X} \boldsymbol{\beta}\right\|^{2}+\lambda_{n} \sum_{j=1}^{p_{n}} \frac{\beta_{j}^{2}}{\tilde{\beta}_{j}^{2}}\right\}=\left\{\mathbf{X}^{\top} \mathbf{X}+\lambda_{n} \mathbf{D}(\tilde{\boldsymbol{\beta}})\right\}^{-1} \mathbf{X}^{\top} \mathbf{Y}^{*}
$$

Finally, the CBAR estimator is defined as

$$
\hat{\boldsymbol{\beta}}^{*}=\lim _{k \rightarrow \infty} \hat{\boldsymbol{\beta}}^{(k)} .
$$

In the next section, we give conditions under which the CBAR estimator $\hat{\boldsymbol{\beta}}^{*}$ is selection consistent and has an oracle property for estimation of the nonzero component $\boldsymbol{\beta}_{01}$ of $\boldsymbol{\beta}$.

\subsection{Large sample properties of CBAR}

Similar to Zhou (1992), define $F_{i}(t)=P\left\{Y_{i} \geq t\right\}, G_{i}(t)=P\left\{T_{i} \geq t\right\}=F_{i}(t)(1-H(t)), K(t)=$ $-\int_{0}^{t} \frac{1}{\lim (1 / n) \sum F_{i}} \frac{d G}{G^{2}}$ and denote

$$
\Lambda_{i}^{+}(t)=-\int_{0}^{t} \frac{d G_{i}(s)}{G_{i}\left(s^{-}\right)}, \Lambda_{i}^{D}(t)=-\int_{0}^{t} \frac{d F_{i}(s)}{F_{i}\left(s^{-}\right)}, \Lambda^{C}(t)=\int_{0}^{t} \frac{d H(s)}{1-H\left(s^{-}\right)} .
$$

Then,

$$
\begin{gathered}
M_{i}^{+}(t)=I_{\left[T_{i} \leq t\right]}-\int_{0}^{t} I_{\left[T_{i} \geq s\right]} d \Lambda_{i}^{+}(s), \\
M_{i}^{D}(t)=I_{\left[T_{i} \leq t ; \delta_{i}=1\right]}-\int_{0}^{t} I_{\left[T_{i} \geq s\right]} d \Lambda_{i}^{D}(s), \\
M_{i}^{C}(t)=I_{\left[T_{i} \leq t ; \delta_{i}=0\right]}-\int_{0}^{t} I_{\left[T_{i} \geq s\right]} d \Lambda_{i}^{C}(s)
\end{gathered}
$$


are square-integrable martingales and satisfies $M_{i}^{+}=M_{i}^{D}+M_{i}^{C}$ (Zhou 1992). Let $\boldsymbol{\Omega}(\tau)=\left(\sigma_{k l}(\tau)\right)$ be defined by

$$
\begin{aligned}
\sigma_{k l}(\tau) & =\lim n \sum_{i=1}^{n} \omega_{k i} \omega_{l i} \int_{0}^{\tau}\left[\int_{t}^{\tau} F_{i} d s\right]^{2} \frac{d \Lambda_{i}^{D}(t)}{G_{i}} \\
& +\lim n \sum_{i=1}^{n} \int_{0}^{\tau} \prod_{c_{i}=\omega_{k i}, \omega_{l i}}\left[\frac{\sum c_{j} \int_{t}^{\tau} F_{j} d s}{(1-H) \sum F_{j}}-\frac{c_{k} \int_{t}^{\tau} F_{i} d s}{G_{i}}\right] G_{i} d \Lambda^{C},
\end{aligned}
$$

where $\omega_{j i}=\left(\left(\mathbf{X}^{\top} \mathbf{X}\right)^{-1} \mathbf{X}^{\top}\right)_{j i}$. Let $\boldsymbol{\omega}_{i}$ denote the $i$ th column of the matrix $\left(\mathbf{X}^{\top} \mathbf{X}\right)^{-1} \mathbf{X}^{\top}, \mathbf{X}_{1}$ denote the first $q_{n}$ columns of $\mathbf{X}, \boldsymbol{\Sigma}_{n}=n^{-1} \mathbf{X}^{\top} \mathbf{X}$ and $\boldsymbol{\Sigma}_{n 1}=n^{-1} \mathbf{X}_{1}^{\top} \mathbf{X}_{1}$. Write $\hat{\boldsymbol{\beta}}^{*}=\left(\hat{\boldsymbol{\beta}}_{1}^{*}, \hat{\boldsymbol{\beta}}_{2}^{*}\right)^{\top}$, where $\hat{\boldsymbol{\beta}}_{1}^{*}$ is a $q \times 1$ vector estimator of $\boldsymbol{\beta}_{01}$ and $\hat{\boldsymbol{\beta}}_{2}^{*}$ is a $\left(p_{n}-q\right) \times 1$ vector estimator of $\boldsymbol{\beta}_{02}$.

The following conditions are needed for our theoretical derivations.

(C1) $\sup _{t} E\left(\varepsilon_{i}-t \mid \varepsilon_{i}>t\right)<\infty$, and for any $p_{n}$-vector $\mathbf{b}_{n}$ satisfying $\left\|\mathbf{b}_{n}\right\| \leq 1, \mathbf{b}_{n}^{\top} \boldsymbol{\Omega}(\tau) \mathbf{b}_{n}$ is finite for $\tau \in[K, \infty]$ and $\mathbf{b}_{n}^{\top} \boldsymbol{\Omega}(\tau) \mathbf{b}_{n} \rightarrow \mathbf{b}_{n}^{\top} \boldsymbol{\Omega}(\infty) \mathbf{b}_{n}$ as $\tau \rightarrow \infty$

$(\mathrm{C} 2) \sup _{n} \int_{0}^{\infty} \sum_{i=1}^{n}\left(\mathbf{b}_{n}^{\top} \boldsymbol{\omega}_{i}\right)^{2} \sum_{i=1}^{n} F_{i}^{2} d K(t)<\infty$ for any $p_{n}$-vector $\mathbf{b}_{n}$ satisfying $\left\|\mathbf{b}_{n}\right\| \leq 1$. $X_{i}$ are bounded, and for some constants $C^{*}>0$ and $S<1, C^{*} F_{i}(t)^{S} \leq 1-H(t)$.

(C3) $\int_{0}^{\infty}\left\{\frac{n \sum\left(\mathbf{b}_{n}^{\top} \boldsymbol{\omega}_{i}\right)^{2} F_{i}}{1-H(s)}\right\}^{\frac{1}{2}} d s \leq M<\infty$ and $\int_{0}^{\infty} K^{1 / 2}(t)\left|\sum \mathbf{b}_{n}^{\top} \boldsymbol{\omega}_{i} F_{i}\right| d t<\infty$ for any $p_{n}$-vector $\mathbf{b}_{n}$ satisfying $\left\|\mathbf{b}_{n}\right\| \leq 1$.

(C4) There exists a constant $\tilde{C}>1$ such that $0<1 / \tilde{C}<\lambda_{\min }\left(\boldsymbol{\Sigma}_{n}\right) \leq \lambda_{\max }\left(\boldsymbol{\Sigma}_{n}\right)<\tilde{C}<\infty$ for every integer $n$.

(C5) Let $a_{0}=\min _{1 \leq j \leq q}\left|\beta_{0 j}\right|$ and $a_{1}=\max _{1 \leq j \leq q}\left|\beta_{0 j}\right|$. As $n \rightarrow \infty, p_{n} / \sqrt{n} \rightarrow 0, \xi_{n} / \sqrt{n} \rightarrow 0$ and $\lambda_{n} / \sqrt{n} \rightarrow 0$

Conditions (C1)-(C3) are regularity conditions required to establish the asymptotic properties of the unpenalized synthetic data least squares estimator under diverging dimension. Conditions (C4) and (C5) are additional conditions needed to derive the selection consistency and oracle property of the synthetic data BAR estimator of this paper as stated in Theorem 1 below

Theorem 1 (Oracle property) Assume conditions (C1)-(C5) hold. For any q-dimensional vector $\mathbf{c}$ satisfying $\|\mathbf{c}\| \leq 1$, define $z^{2}=\mathbf{c}^{\top} \boldsymbol{\Omega}_{1} \mathbf{c}$, where $\boldsymbol{\Omega}_{1}$ is the first $q \times q$ sub-matrix of $\boldsymbol{\Omega}(\infty)$. Define $f(\boldsymbol{\alpha})=$ $\left\{\mathbf{X}_{1}^{\top} \mathbf{X}_{1}+\lambda_{n} \mathbf{D}_{1}(\boldsymbol{\alpha})\right\}^{-1} \mathbf{X}_{1}^{\top} \mathbf{Y}^{*}$, where $\mathbf{D}_{1}(\boldsymbol{\alpha})=\operatorname{diag}\left(\alpha_{1}^{-2}, \ldots, \alpha_{q}^{-2}\right)$. Then, with probability tending to 1 ,

(i) $\widehat{\boldsymbol{\beta}}^{*}=\left(\widehat{\boldsymbol{\beta}}_{1}^{*}, \widehat{\boldsymbol{\beta}}_{2}^{* \top}\right)^{\top}$ exists and is unique, with $\hat{\boldsymbol{\beta}}_{2}^{*}=0$ and $\hat{\boldsymbol{\beta}}_{1}^{*}$ being the unique fixed point of $f(\boldsymbol{\alpha})$;

(ii) $\sqrt{n} z^{-1} \mathbf{c}^{\top}\left(\hat{\boldsymbol{\beta}}_{1}^{*}-\boldsymbol{\beta}_{01}\right) \rightarrow_{D} N(0,1)$.

Part (i) of the above theorem guarantees that the CBAR estimator is consistent for variable selection. Part (ii) states that the asymptotic distribution of the nonzero component of the CBAR estimator is the same as the one when the true model is known in advance. The proof of Theorem 1 is deferred to the Appendix.

\subsection{Grouping effect}

When the true model has a group structure, it would be desirable for a variable selection method to either retain or drop all variables that are clustered within the same group. Below we establish that the CBAR estimator possesses a grouping property in the sense that highly correlated covariates tend to be grouped together with similar coefficients. 
Theorem 2 Assume that the columns of matrix $\mathbf{X}$ are standardized and $\mathbf{Y}^{*}$ is centered. Let $\hat{\boldsymbol{\beta}}^{*}$ be the $C B A R$ estimator and $\hat{\beta}_{i}^{*} \hat{\beta}_{j}^{*}>0$, then, with probability tending to 1 ,

$$
\left|\hat{\beta}_{i}^{*-1}-\hat{\beta}_{j}^{*-1}\right| \leq \frac{1}{\lambda_{n}}\left\|\mathbf{Y}^{*}\right\| \sqrt{2\left(1-r_{i j}\right)}
$$

where $r_{i j}=\mathbf{x}_{i}^{\top} \mathbf{x}_{j}$ is the sample correlation of $\mathbf{x}_{i}$ and $\mathbf{x}_{j}$.

The above result implies that the estimated coefficients of two highly positively-correlated variables will be similar in magnitude. The proof of Theorem 2 is given in the Appendix. Similarly, it can be shown that the estimated coefficients of two highly negatively-correlated variables will also be similar in magnitude.

\subsection{Ultrahigh dimensional covariates}

Theorem 1 is established under a sufficient condition that $p_{n}<n$. In many applications, $p_{n}$ can be much larger than the sample size $n$. For high dimensional problems, a common strategy is to proceed a variable selection method with a sure screening dimension reduction step (Fan and Lv 2008; Zhu et al. 2011; Cui et al. 2015). This strategy also applies to the semiparametric AFT model with right censored data. For example, one can first apply the sure joint screening method BJASS of Liu et al. (2019) to obtain a lower dimensional model and then apply the CBAR method to the reduced model. We refer to the resulting two-step estimator $\hat{\boldsymbol{\beta}}^{*}$ as the BJASS-CBAR estimator.

Below we give some additional sufficient conditions under which the BJASS-CBAR estimator $\hat{\boldsymbol{\beta}}^{*}$ has an oracle property.

(D1) $\log (p)=O\left(n^{d}\right)$ for some $0 \leq d<1$.

(D2) $P\left(t \leq Y_{i} \leq C_{i}\right) \geq \tau_{0}>0$ for some positive constant $\tau_{0}$ and any $t \in[0, \varsigma]$, where $\varsigma$ denotes the maximum follow up time. Furthermore, $\sup \{t: P(Y>t)>0\} \geq \sup \{t: P(C>t)>0\}$. H(t) has uniformly bounded first derivative.

(D3) $\min _{j \in s^{*}}\left|\beta_{j}^{*}\right| \geq \omega_{1} n^{-\tau_{1}}$ and $q<k \leq \omega_{2} n^{\tau_{2}}$ for some positive constants $\omega_{1}$, $\omega_{2}$ and nonnegative constants $\tau_{1}, \tau_{2}$ satisfying $\tau_{1}+\tau_{2}<1 / 3$, where $k$ is the size of the screened model from BJASS.

(D4) For sufficiently large $n, \lambda_{\min }\left(n^{-1} \mathbf{X}_{s}^{\top} \mathbf{X}_{s}\right) \geq c_{1}$ for some constant $c_{1}>0$ and all $s \in S_{+}^{2 k}$, where $\lambda_{\min }(\cdot)$ denotes the smallest eigenvalue of a matrix, and $S_{+}^{k}=\left\{s: s^{*} \subset s ;\|s\|_{0} \leq k\right\}$ denotes the collection of the over-fitted models of cardinality $k$ or smaller.

(D5) Let $\sigma_{i}^{2}=\iint\left[\frac{G_{i}(s \vee t)}{(1-H(s))(1-H(t))}-F_{i}(s) I(t<0)-F_{i}(t) I(s<0)+I(s<0) I(t<0)\right] d s d t-E^{2}\left(Y_{i}\right)$. There exist positive constants $c_{2}, c_{3}, c_{4}, \sigma$ such that $\left|X_{i j}\right| \leq c_{2},\left|X_{i}^{\top} \boldsymbol{\beta}^{*}\right| \leq c_{3},\left|\sigma_{i}\right| \leq \sigma$ and for sufficiently large $n$,

$$
\max _{1 \leq j \leq p} \max _{1 \leq i \leq n}\left\{\frac{X_{i j}^{2}}{\sum_{i=1}^{n} X_{i j}^{2} \sigma_{i}^{2}}\right\} \leq c_{4} n^{-1} .
$$

(D6) There are positive constants $K_{1}, K_{2}$ and $\tau_{3}$ such that

$$
P(|\epsilon| \geq M) \leq K_{1} \exp \left(-K_{2} M^{\tau_{3}}\right)
$$

for any $M=O\left(n^{\tau}\right)>0$, where $\tau \geq 0, \tau_{1}+\tau_{2}+\tau<(1-d) / 2, \tau_{2}+d-\tau \tau_{3}<0$, and $2 \tau_{2}+2 \tau+d<1 / 3$.

Theorem 3 (Oracle property of the BJASS-CBAR estimator) Assume that conditions (D1)-(D6) hold and that the assumptions of Theorem 1 hold for the BJASS reduced model. Then, with probability tending to 1 , 
(i) $\hat{\boldsymbol{\beta}}_{2}^{*}=0$;

(ii) $\hat{\boldsymbol{\beta}}_{1}^{*}$ performs as well as the oracle estimator for the true model $\mathcal{M}_{*}=\{1 \leq j \leq q\}$ in the sense of part (ii) of Theorem 1.

The above result is a direct consequence of Theorems 4 of Liu et al. (2019) and the oracle property of CBAR stated in Theorem 1. In Section 3.2, we present a simulation study to illustrate the advantages of BJASS-BAR in comparison with some other penalization methods under a high dimensional setting.

\section{Simulations}

We present some simulations to illustrate the effectiveness of the proposed CBAR estimator for variable selection, prediction, parameter estimation in comparison with some popular penalization methods including Lasso (Tibshirani 1996), adaptive Lasso (Zou 2006), SCAD (Fan and Li 2001) and MCP (Zhang 2010)), in the context of the Leurgans (1987) synthetic data framework. We use the R package glmnet (Friedman et al. 2010) for Lasso and adaptive Lasso and R package ncvreg (Breheny and Huang 2011) for SCAD and MCP, performed on the Leurgans (1987) synthetic data outcome. Five-fold crossvalidation $(\mathrm{CV})$ is used to select tuning parameters for all methods. For CBAR, we all use 10 equally log-spaced grid points on $[a, b]$ for the paths of $\lambda_{n}$ and $\xi_{n}$ where $a=1 e^{-4}$ and $b=\max \left\{\frac{\left(\mathbf{x}_{j}^{\mathrm{T}} \mathbf{y}\right)^{2}}{4 \mathbf{x}_{j}^{\mathrm{T}} \mathbf{x}_{j}}\right\}_{j=1}^{p}$.

\subsection{Simulation 1: $p_{n}<n$}

We consider the following two model settings similar to (Tibshirani 1997; Fan and Li 2002; Cai et al. 2009):

Model 1: $Y_{i}=\mathbf{x}_{i}^{\top} \boldsymbol{\beta}_{0}+\varepsilon_{i}$, where the covariate vector $\mathbf{x}_{i}$ is generated from a multivariate normal distribution with mean 0 and variance-covariance matrix $\boldsymbol{\Sigma}=\left(\rho^{|i-j|}\right)$, and the error $\varepsilon_{i}$ has the standard normal distribution and is independent of the covariates.

The true parameter value is $\boldsymbol{\beta}_{0}=(3,-2,0,0,6,0, \ldots, 0)^{\top}$.

Model 2: The same as Model 1 except that

$$
\boldsymbol{\beta}_{0}=(3,-2,6,0.3,-0.2,0.6,0, \ldots, 0)^{\top} \text {. }
$$

Note that Model 1 contains strong signals, whereas Model 2 includes both strong and weak signals. The censoring variable $C_{i}$ is generated from the normal distribution $N(c, 2)$, where $c$ is chosen to yield a desired level of censoring rate.

The variable selection performance is assessed using five measures: the mean number of misclassified non-zeros and zeros (MisC), mean of false non-zeros (FP), mean of false zeros (FN), probability that the selected model is identical to the true model (TM), and a similarity measure (SM) between the selected set $\hat{S}$ and the true active set $|S|_{0}: S M=\frac{|\hat{S} \cap S|_{0}}{\sqrt{|\hat{S}|_{0}|S|_{0}}}$, where $|\cdot|_{0}$ denotes model size. The prediction performance is measured by the mean squared prediction error (MSPE) from the five-fold $\mathrm{CV}$. The parameter estimation performance is measured by the mean of the absolute bias of the parameter estimator (MAB). We have run extensive simulations for a variety of settings by varying $n$, $p, \rho$ and the censoring rate, with 1,000 Monte Carlo replications for each setting. Part of the findings are presented in Table 1.

[Insert Table 1 approximately here] 
Table 1 Comparison of CBAR with Lasso, SCAD, MCP, and Adaptive Lasso (ALasso) when coupled with the Leurgans (1987) synthetic data procedure based on 1,000 Monte-Carlo replications. Data settings: $n=100, p \in$ $\{10,50,80,90\}$. (MisC $=$ mean number of misclassified non-zeros and zeros; FP $=$ mean of false positives (nonzeros); FN = mean of false negatives (zeros); $\mathrm{TM}=$ probability that the selected model is exactly the true model; $\mathrm{SM}=$ similarity measure; $\mathrm{MSPE}=$ mean squared prediction error from five-fold CV or five-jointly CV and $\mathrm{MAB}=$ mean of the absolute bias of the parameter estimator.)

\begin{tabular}{|c|c|c|c|c|c|c|c|c|c|}
\hline "Model & $\bar{p}$ & "Method & MisC & $\overline{\mathrm{FP}}$ & $\overline{\mathrm{FN}}$ & $\overline{T \text { TM }}$ & $\overline{\mathrm{SM}}$ & "MSPE & $\overline{\mathrm{MAB}}$ \\
\hline \multirow[t]{20}{*}{1} & \multirow[t]{5}{*}{10} & CBAR & 0.60 & 0.60 & 0 & $74 \%$ & 0.94 & 8.86 & 1.50 \\
\hline & & Lasso & 3.05 & 3.05 & 0 & $6.6 \%$ & 0.73 & 9.28 & 2.29 \\
\hline & & SCAD & 1.11 & 1.11 & 0 & $46.2 \%$ & 0.89 & 9.03 & 1.47 \\
\hline & & MCP & 0.76 & 0.76 & 0 & $63.6 \%$ & 0.92 & 9.01 & 1.45 \\
\hline & & Alasso & 1.12 & 1.12 & 0 & $49.2 \%$ & 0.89 & 8.91 & 1.68 \\
\hline & \multirow[t]{5}{*}{50} & CBAR & 0.73 & 0.71 & 0.02 & $74.80 \%$ & 0.94 & 8.9 & 1.69 \\
\hline & & Lasso & 7.33 & 7.33 & 0 & $1.7 \%$ & 0.58 & 9.77 & 3.36 \\
\hline & & SCAD & 2.96 & 2.96 & 0 & $21.3 \%$ & 0.76 & 9.09 & 1.72 \\
\hline & & MCP & 1.24 & 1.23 & 0.01 & $47.7 \%$ & 0.88 & 9.03 & 1.56 \\
\hline & & Alasso & 6.09 & 6.09 & 0 & $15.2 \%$ & 0.67 & 8.69 & 3.04 \\
\hline & \multirow[t]{5}{*}{80} & CBAR & 0.86 & 0.84 & 0.02 & $72.3 \%$ & 0.93 & 8.84 & 1.81 \\
\hline & & Lasso & 9.40 & 9.40 & 0 & $1.30 \%$ & 0.54 & 10.06 & 3.79 \\
\hline & & SCAD & 3.90 & 3.90 & 0 & $15.2 \%$ & 0.72 & 9.33 & 1.89 \\
\hline & & MCP & 1.41 & 1.40 & 0.01 & $45.6 \%$ & 0.87 & 9.26 & 1.65 \\
\hline & & Alasso & 11.09 & 11.08 & 0.01 & $11.8 \%$ & 0.59 & 8.74 & 4.51 \\
\hline & \multirow[t]{5}{*}{90} & CBAR & 0.94 & 0.92 & 0.02 & $69.7 \%$ & 0.93 & 8.93 & 1.88 \\
\hline & & Lasso & 9.36 & 9.36 & 0 & $1.4 \%$ & 0.54 & 10.05 & 3.82 \\
\hline & & SCAD & 4.09 & 4.09 & 0 & $13.5 \%$ & 0.71 & 9.27 & 1.91 \\
\hline & & MCP & 1.44 & 1.43 & 0.01 & $43.2 \%$ & 0.87 & 9.20 & 1.64 \\
\hline & & Alasso & 4.29 & 4.27 & 0.02 & $10.5 \%$ & 0.70 & 9.13 & 2.69 \\
\hline \multirow[t]{20}{*}{2} & \multirow[t]{5}{*}{10} & CBAR & 2.61 & 0.65 & 1.96 & $0.9 \%$ & 0.77 & 9.36 & 2.36 \\
\hline & & Lasso & 3.00 & 2.14 & 0.86 & $2.2 \%$ & 0.78 & 9.50 & 2.74 \\
\hline & & SCAD & 2.64 & 1.10 & 1.54 & $2.3 \%$ & 0.78 & 9.35 & 2.35 \\
\hline & & MCP & 2.64 & 0.86 & 1.78 & $1.9 \%$ & 0.77 & 9.34 & 2.36 \\
\hline & & Alasso & 2.50 & 0.92 & 1.58 & $3.1 \%$ & 0.79 & 9.14 & 2.37 \\
\hline & \multirow[t]{5}{*}{50} & CBAR & 3.65 & 1.03 & 2.62 & $0.1 \%$ & 0.69 & 9.41 & 2.92 \\
\hline & & Lasso & 9.75 & 7.99 & 1.76 & $0 \%$ & 0.52 & 10.40 & 4.37 \\
\hline & & SCAD & 5.57 & 3.40 & 2.17 & $0 \%$ & 0.61 & 9.84 & 2.77 \\
\hline & & MCP & 3.92 & 1.46 & 2.46 & $0 \%$ & 0.67 & 9.80 & 2.64 \\
\hline & & Alasso & 9.18 & 7.22 & 1.96 & $0.1 \%$ & 0.55 & 9.21 & 4.27 \\
\hline & \multirow[t]{5}{*}{80} & CBAR & 3.89 & 1.19 & 2.70 & $0 \%$ & 0.68 & 9.11 & 3.02 \\
\hline & & Lasso & 11.61 & 9.69 & 1.92 & $0 \%$ & 0.48 & 10.39 & 4.70 \\
\hline & & SCAD & 6.48 & 4.21 & 2.27 & $0 \%$ & 0.57 & 9.66 & 2.86 \\
\hline & & MCP & 4.06 & 1.49 & 2.57 & $0 \%$ & 0.66 & 9.60 & 2.64 \\
\hline & & Alasso & 13.82 & 11.78 & 2.04 & $0 \%$ & 0.48 & 8.99 & 5.55 \\
\hline & \multirow[t]{5}{*}{90} & CBAR & 3.85 & 1.16 & 2.69 & $0 \%$ & 0.68 & 9.31 & 3.05 \\
\hline & & Lasso & 12.44 & 10.47 & 1.97 & $0 \%$ & 0.46 & 10.20 & 4.86 \\
\hline & & SCAD & 6.92 & 4.67 & 2.25 & $0 \%$ & 0.56 & 9.40 & 2.92 \\
\hline & & MCP & 4.24 & 1.68 & 2.56 & $0 \%$ & 0.65 & 9.36 & 2.68 \\
\hline & & Alasso & 7.00 & 4.50 & 2.50 & $0 \%$ & 0.54 & 9.27 & 3.73 \\
\hline
\end{tabular}

It is seen from Table 1 that CBAR stands out as the top or top two performers with respect to almost all variable selection performance measures (MisC, FP, TM and SM). In particular, CBAR generally yields a more sparse and accurate model with the largest TM and SM, and much lower MisC and FP. Also, using fewer active features, CBAR achieves comparable prediction accuracy as other methods that use more features. For estimation, CBAR, SCAD and MCP are comparable with similar bias (MAB), whereas Lasso and Adaptive lasso can be substantially worse.

\subsection{Simulation 2: $p_{n}>>n$}

In this simulation, we consider the same models as in Simulation 1, except in a high dimensional setting with $n=200, p=1000$. We again compared the same five penalization methods, with each method proceeded with the sure joint screening method BJASS of Liu et al. (2019) with $k=2 \log (n) * n^{(1 / 4)}$ for the semi-parametric AFT model to yield a two-step sparse estimator. We denote these methods by BJASS-CBAR, BJASS-Lasso, BJASS-SCAD, BJASS-MCP and BJASS-ALasso. The censoring rate is 0.2 . The results are summarized in Table 2 . 
[Insert Table 2 approximately here]

Table 2 Comparison of BJASS-CBAR with CBAR with BJASS-Lasso, BJASS-SCAD, BJASS-MCP, and BJASSALasso when coupled with the Leurgans (1987) synthetic data procedure in a high-dimensional setting: $n=200$, $p=1000$. (MisC = mean number of misclassified non-zeros and zeros; $\mathrm{FP}=$ mean of false positives (non-zeros) $\mathrm{FN}=$ mean of false negatives (zeros); $\mathrm{TM}=$ probability that the selected model is exactly the true model; $\mathrm{SM}=$ similarity measures; $\mathrm{MSPE}=$ mean squared prediction error from five-fold CV or five-jointly CV and $\mathrm{MAB}=$ mean of the absolute bias of the parameter estimator.)

\begin{tabular}{cllllllll}
\hline Model & Method & MisC & FP & FN & TM & SM & MAB & MSPE \\
\hline 1 & BJASS-CBAR & $\mathbf{2 . 2 4}$ & $\mathbf{2 . 1 5}$ & 0.09 & $\mathbf{6 3} \%$ & $\mathbf{0 . 9 3}$ & 2.87 & 10.40 \\
& BJASS-Lasso & 12.61 & 12.55 & 0.06 & $0 \%$ & 0.63 & 4.79 & 10.87 \\
& BJASS-SCAD & 4.23 & 4.14 & 0.09 & $20 \%$ & 0.82 & 2.79 & 10.46 \\
& BJASS-MCP & 2.82 & 2.73 & 0.09 & $43 \%$ & 0.88 & $\mathbf{2 . 6 9}$ & 10.45 \\
& BJASS-ALasso & 8.08 & 8.00 & 0.08 & $12 \%$ & 0.73 & 4.05 & 10.35 \\
\hline \multirow{2}{*}{2} & BJASS-CBAR & $\mathbf{6 . 1 5}$ & $\mathbf{3 . 1 5}$ & 3 & $\mathbf{4 1} \%$ & $\mathbf{0 . 6 9}$ & 2.51 & 12.17 \\
& BJASS-Lasso & 17.14 & 14.14 & 3 & $0 \%$ & 0.49 & 4.49 & 12.64 \\
& BJASS-SCAD & 8.68 & 5.68 & 3 & $7 \%$ & 0.62 & 2.09 & 12.39 \\
& BJASS-MCP & 6.38 & 3.38 & 3 & $26 \%$ & 0.68 & $\mathbf{1 . 9 6}$ & 12.38 \\
& BJASS-ALasso & 12.78 & 9.78 & 3 & $3 \%$ & 0.54 & 3.75 & 11.91 \\
\hline
\end{tabular}

It is observed from Table 2 that although most penalization methods had comparable performance in terms of estimation bias (MAB) and prediction error (MSPE), BJASS-CBAR outperformed the other methods in the variable selection domain with the lowest MisC, FP and the largest TM and SM, which are consistent with the simulation results for the low-dimension $p_{n}<n$ settings in Simulation 1 .

\section{Real data examples}

We illustrate the CBAR method on two real datasets with high dimensional covariates.

4.1 Diffuse large-B-cell lymphoma data

The diffuse large-B-cell lymphoma (DLBCL) data includes $n=240$ patients and $p=7399$ gene features, which was downloaded from http://statweb.stanford.edu/ tibs/superpc/staudt.html. We first apply the BJASS sure joint screening method of Liu et al. (2019) to reduce data dimension to $k=2 \log (n) n^{\frac{1}{4}}=43$ and then apply CBAR and four other popular penalization methods. The results are summarized in Table 3.

[Insert Table 3 approximately here]

It is seen that BJASS-CBAR is among the most sparse model and has the smallest CV error, which is consistent with the findings in the simulation studies.

\subsection{Glioblastoma multiforme data}

The glioblastoma multiforme (GBM) methylation data was downloaded from the TCGA program (https://www.cancer.gov/tcga) using TCGA-Assembler 2 (TA2). The initial data consists of 577 patients and 20,156 GBM methylation variables. After removing missing data, the complete case data 
Table 3 Estimated coefficients of BAJSS-CBAR, BAJSS-Lasso, BAJSS-SCAD, BAJSS-MCP and BAJSS-Alasso for the DLBCL data.

\begin{tabular}{|c|c|c|c|c|c|}
\hline Parameter & BAJSS-CBAR & BAJSS-Lasso & BAJSS-SCAD & BAJSS-MCP & BAJSS-Alasso \\
\hline 1456 & -0.0591 & -0.394 & -0.609 & -0.630 & $\overline{-0.513}$ \\
\hline 1819 & & -0.069 & & & \\
\hline 1863 & & -0.006 & & & \\
\hline 2603 & & -0.025 & & & \\
\hline 2672 & & -0.062 & & & \\
\hline 3236 & -0.480 & -0.348 & -0.394 & -0.426 & -0.399 \\
\hline 5775 & -0.261 & -0.143 & -0.133 & -0.131 & -0.111 \\
\hline 6566 & & -0.088 & -0.061 & -0.004 & \\
\hline Tuning parameters & $\begin{array}{c}\xi_{n}=43 \\
\lambda_{n}=5.721\end{array}$ & $\lambda=0.197$ & $\begin{array}{c}\gamma=3.7 \\
\lambda=0.211\end{array}$ & $\lambda=0.260$ & $\begin{array}{l}\gamma=3.598, \\
\lambda=2.058\end{array}$ \\
\hline Number of selected & 3 & 8 & 4 & 4 & 3 \\
\hline CV error & 6.399 & 6.731 & 6.496 & 6.515 & 6.472 \\
\hline
\end{tabular}

includes $n=136$ patients and $p=20,037$ methylation variables. Applying the method described in Section 2.5, we first performed sure joint screening using the BJASS method of Liu et al. (2019) reduce data dimension to $k=2 \log (n) n^{\frac{1}{4}}=34$ before applying the CBAR penalization method and four other penalization methods (Lasso, SCAD, MCP and Alasso). The final variable selection results are summarized in the Table 4.

[Insert Table 4 approximately here]

Table 4 Estimated coefficients of BJASS-CBAR, BJASS-Lasso, BJASS-SCAD, BJASS-MCP and BJASS-Alasso for the TCGA GBM methylation data

\begin{tabular}{|c|c|c|c|c|c|}
\hline Variables & BJASS-CBAR & BJASS-Lasso & BJASS-SCAD & BJASS-MCP & BJASS-Alasso \\
\hline BCL2L10 & & 0.051 & 0.038 & & 0.038 \\
\hline CDCP2 & -0.272 & -0.077 & -0.057 & & -0.068 \\
\hline HES5 & & -0.139 & -0.153 & -0.265 & -0.162 \\
\hline HLA.E & & 0.104 & 0.117 & 0.167 & 0.098 \\
\hline HRH3 & & 0.021 & & & \\
\hline IRX 6 & & 0.014 & & & \\
\hline KIF5C & & 0.004 & & & \\
\hline NIPSNAP3B & & 0.034 & & & 0.017 \\
\hline NPM2 & 0.230 & 0.087 & 0.065 & 0.089 & 0.078 \\
\hline OXGR1 & & 0.059 & 0.066 & & 0.045 \\
\hline SLC12A5 & 0.282 & 0.144 & 0.104 & 0.072 & 0.167 \\
\hline SMIM11A & 0.417 & 0.349 & 0.469 & 0.507 & 0.418 \\
\hline Tuning parameters & $\begin{array}{c}\xi_{n}=19 \\
\lambda_{n}=1.642\end{array}$ & $\lambda=0.122$ & $\begin{array}{c}\gamma=3.7 \\
\lambda=0.154\end{array}$ & $\lambda=0.190$ & $\lambda=0.625$ \\
\hline Number of selected & 4 & 12 & 9 & 5 & 9 \\
\hline CV error & 3.793 & 3.832 & 3.804 & 3.835 & 3.620 \\
\hline
\end{tabular}

It is seen from Table 4 that our BJASS-CBAR selected the sparsest model with 4 variables while achieving a comparable CV error as compared to the other four methods, which is consistent with our findings in simulation studies. It is interesting to note that the four features selected by BJASS-CBAR have also been selected by three other methods. Among the four selected features, NPM2 and IRX6 have been previously discussed in the literature to possibly play critical roles with human diseases (Eirin-Lopez and J. 2006; Box 2016; Nachmani et al. 2019; MUM 2001). 


\section{Discussion}

We have rigorously extended the broken adaptive ridge (BAR) penalization method for simultaneous variable selection and parameter estimation to the semiparametric AFT model with right-censored data by coupling BAR penalization with the Leurgans (1987) synthetic data. We have established that the resulting CBAR estimator is asymptotically consistency for variable selection, has an oracle estimation property, and enjoys a grouping property for highly correlated covariates. We consider both low and high dimensional covariate settings. Our empirical studies demonstrate that CBAR generally produces a more sparse and accurate model as compared to some popular $L_{1}$-based penalization methods, which corroborates previous findings in the literature for uncensored data.

We note that coupling the BAR method with the Leurgans (1987) synthetic variable is only one of several possible ways of extending the BAR method to right censored linear model for simultaneous variable selection and parameter estimation. For example, one may couple the BAR method with the Koul et al. (1981) synthetic data method, the Stute (1993) weighted least squares method, or the Buckley and James (1979) iterative imputation method. Our limited numerical studies (not reported here) indicate that using Koul et al. (1981) synthetic data is generally inferior to using Leurgans (1987) synthetic variable, whereas iteratively performing BAR using the Buckley and James (1979) imputation may sometimes improve the performance of the CBAR method based on the Leurgans (1987) synthetic variable. However, asymptotic properties of each of these distinct approaches require different theoretical developments. Thorough investigations and comparisons of these alternative approaches are needed in future research.

Lastly, missing data often occurs in real world applications. Although there is a vast amount literature on missing data problems, little has been done to deal with missing data in the context of variable selection for survival data. Further research in this domain is waranteed.

\section{Acknowledgement}

The Glioblastoma multiforme data used in Section 4.2 are generated by the TCGA Research Network: https://www.cancer.gov/tcga.

\section{References}

Expression of irx6 during mouse morphogenesis. Mechanisms of Development, 103(1):193 - 195, 2001. ISSN 09254773.

Nucleophosmin: from structure and function to disease development. Bmc Molecular Biology, 17(1), 2016.

H. Akaike. A new look at the statistical model identification. IEEE Trans. Automat. Contr., 19:716-723., 1974.

P. Breheny and J. Huang. Coordinate descent algorithms for nonconvex penalized regression, with applications to biological feature selection. The annals of applied statistics, 5(1):232-253., 2011.

L. Breiman. Heuristics of instability and stabilization in model selection. Ann. Statist., 24:2350-2383, 1996.

J. Buckley and I. James. Linear regression with censored data. Biometrika, 66(3):429-436, 1979.

T. Cai, J. Huang, and L. Tian. Regularized estimation for the accelerated failure time model. Biometrics, 65(2): 394-404, 2009.

J. Chen and Z. Chen. Extended bayesian information criteria for model selection with large model spaces. Biometrika, 95:759-771, 2008.

B. D. R. Cox. Regression models and life-tables. Journal of the Royal Statistical Society: Series B (Methodological), 34(2):187-220., 1972.

H. Cui, R. Li, and W. Zhong. Model-free feature screening for ultrahigh dimensional discriminant analysis. Journal of the American Statistical Association, 110(510):630-641, 2015.

L. Dai, K. Chen, and G. Li. The broken adaptive ridge procedure and its applications. Stat Sin, 2018a. doi: $10.5705 /$ ss.202018.0075. 
L. Dai, K. Chen, Z. Sun, Z. Liu, and G. Li. Broken adaptive ridge regression and its asymptotic properties. Journal of Multivariate Analysis, 168:334-351, 2018b.

S. Datta, J. Le-Rademacher, and S. Datta. Predicting patient survival from microarray data by accelerated failure time modeling using partial least squares and lasso. Biometrics, 63(1), 2007.

Eirin-Lopez and M. J. Long-term evolution and functional diversification in the members of the nucleophosmin/nucleoplasmin family of nuclear chaperones. Genetics, 173(4):1835-50, 2006.

J. Fan and R. Li. Variable selection via nonconcave penalized likelihood and its oracle properties. Journal of the American statistical Association, 96(456):1348-1360, 2001.

J. Fan and R. Li. Variable selection for cox's proportional hazards model and frailty model. The Annals of Statistics, 30(1):74-99, 2002.

J. Fan and J. Lv. Sure independence screening for ultrahigh dimensional feature space. Journal of the Royal Statistical Society, 70(5):849-911, 2008.

D. Foster and E. George. The risk inflation criterion for multiple regression. Ann. Statist., 22:1947-1975, 1994.

J. Friedman, T. Hastie, and R. Tibshirani. Regularization paths for generalized linear models via coordinate descent. Journal of statistical software, 33(1):1-22, 2010.

J. Huang and S. Ma. Variable selection in the accelerated failure time model via the bridge method. Lifetime Data Analysis, 16(2):176-95, 2010

J. Huang, S. Ma, and H. Xie. Regularized estimation in the accelerated failure time model with high-dimensional covariates. Biometrics, 62(3):813-820, 2006.

B. A. Johnson. On lasso for censored data. Electronic Journal of Statistics, 3(2009):485-506, 2009.

B. A. Johnson, D. Y. Lin, and D. Zeng. Penalized estimating functions and variable selection in semiparametric regression models. Journal of the American Statistical Association, 103(482):672-680, 2008.

J. D. Kalbfleisch and R. L. Prentice. The Statistical Analysis of Failure Time Data, 2nd Edition. 2002.

E. S. Kawaguchi, M. A. Suchard, Z. Liu, and G. Li. A surrogate 10 sparse cox's regression with applications to sparse high-dimensional massive sample size time-to-event data. Statistics in Medicine, n/a(n/a), 2019. doi: 10.1002/sim.8438. URL https://onlinelibrary.wiley.com/doi/abs/10.1002/sim.8438.

H. Koul, V. Susarla, and J. V. Ryzin. Regression analysis with randomly right-censored data. Annals of Statistics, 9(6):1276-1288, 1981.

S. Leurgans. Linear models, random censoring and synthetic data. Biometrika, 74(2):301-309, 1987.

D. Lin, D. P. Foster, and L. H. Ungar. A risk ratio comparison of 10 and 11 penalized regressions. University of Pennsylvania, techical report, 2010.

Y. Liu, X. Chen, and G. Li. A new joint screening method for right-censored time-to-event data with ultra-high dimensional covariates. Statistical Methods in Medical Research, 2019. doi: 10.1177/0962280219864710.

C. Mallows. Some comments on $c_{p}$. Technometrics, 15:661-675, 1973.

D. Nachmani, A. H. Bothmer, S. Grisendi, A. Mele, and P. P. Pandolfi. Nature Genetics, 51(10):1518-1529, 2019.

G. Schwarz. Estimating the dimension of a model. Ann. Statist., 6:461-464, 1978.

X. Shen, W. Pan, and Y. Zhu. Likelihood-based selection and sharp parameter estimation. J. Amer. Statist. Assoc., 107:223-232, 2012.

W. Stute. Consistent estimation under random censorship when covariables are present. Journal of Multivariate Analysis, 45(1):89-103., 1993.

R. Tibshirani. Regression shrinkage and selection via the lasso. Journal of the Royal Statistical Society. Series B (Methodological), 58(1):267-288, 1996.

R. Tibshirani. The lasso method for variable selection in the cox model. Statistics in Medicine, 16(4):385-395, 1997.

S. Wang, B. Nan, J. Zhu, and D. G. Beer. Biometrics, 64(1):132-40, 2008.

Yi and Li. Dantzig selector for censored linear regression models:with applications in high dimensional data analysis. In International Conference on Financial Statistics and Financial Econometrics, 2009.

C.-H. Zhang. Nearly unbiased variable selection under minimax concave penalty. The Annals of statistics, 38(2): 894-942, 2010.

H. Zhao, Q. Wu, G. Li, and J. Sun. Simultaneous estimation and variable selection for interval-censored data with broken adaptive ridge regression. Journal of the American Statistical Association, 0(0):1-13, 2019. doi: 10.1080/01621459.2018.1537922. URL https://doi.org/10.1080/01621459.2018.1537922.

M. Zhou. Asymptotic normality of the synthetic data regression estimator for censored survival data. Annals of Statistics, 20(2):1002-1021, 1992.

L. Zhu, L. Li, R. Li, and L. Zhu. Model-free feature screening for ultrahigh dimensional data. Publications of the American Statistical Association, 106(496):1464-1475, 2011.

H. Zou. The adaptive lasso and its oracle properties. Journal of the American statistical association, 101(476): 1418-1429, 2006. 


\section{A Proofs of the theorem}

We first introduce notations and lemmas used to prove Theorem 1.

Using Leurgans (1987) method, we transform $\mathbf{Y}$ into synthetic data $\mathbf{Y}^{*}$. Let $\boldsymbol{\beta}=\left(\boldsymbol{\alpha}^{\top}, \boldsymbol{\gamma}^{\top}\right)^{\top}$, where $\boldsymbol{\alpha}$ and $\boldsymbol{\gamma}$ are $q_{n} \times 1$ and $\left(p_{n}-q_{n}\right) \times 1$ vector respectively, $\boldsymbol{\Sigma}_{n}=\mathbf{X}^{\top} \mathbf{X} / n$.

$$
g(\boldsymbol{\beta})=\left\{\mathbf{X}^{\top} \mathbf{X}+\lambda_{n} \mathbf{D}(\boldsymbol{\beta})\right\}^{-1} \mathbf{X}^{\top} \mathbf{Y}^{*}=\left(\boldsymbol{\alpha}^{*}(\boldsymbol{\beta})^{\top}, \boldsymbol{\gamma}^{*}(\boldsymbol{\beta})^{\top}\right)^{\top} .
$$

For simplicity, we write $\boldsymbol{\alpha}^{*}(\boldsymbol{\beta})$ and $\boldsymbol{\gamma}^{*}(\boldsymbol{\beta})$ as $\boldsymbol{\alpha}^{*}$ and $\boldsymbol{\gamma}^{*}$ hereafter. $\boldsymbol{\Sigma}_{n}^{-1}$ can be partitioned as

$$
\boldsymbol{\Sigma}_{n}^{-1}=\left(\begin{array}{ll}
\mathbf{A}_{11} & \mathbf{A}_{12} \\
\mathbf{A}_{12}^{\top} & \mathbf{A}_{22}
\end{array}\right)
$$

where the $A_{11}$ is a $q \times q$ matrix. Multiplying $\left(\mathbf{X}^{\top} \mathbf{X}\right)^{-1}\left(\mathbf{X}^{\top} \mathbf{X}+\lambda_{n} \mathbf{D}(\boldsymbol{\beta})\right)$ to equation (10)

$$
\left(\begin{array}{c}
\boldsymbol{\alpha}^{*}-\boldsymbol{\beta}_{01} \\
\boldsymbol{\gamma}^{*}
\end{array}\right)+\frac{\lambda_{n}}{n}\left(\begin{array}{c}
\mathbf{A}_{11} \mathbf{D}_{1}(\boldsymbol{\alpha}) \boldsymbol{\alpha}^{*}+\mathbf{A}_{12} \mathbf{D}_{2}(\boldsymbol{\gamma}) \boldsymbol{\gamma}^{*} \\
\mathbf{A}_{12}^{\top} \mathbf{D}_{1}(\boldsymbol{\alpha}) \boldsymbol{\alpha}^{*}+\mathbf{A}_{22} \mathbf{D}_{2}(\boldsymbol{\gamma}) \boldsymbol{\gamma}^{*}
\end{array}\right)=\left(\mathbf{X}^{\top} \mathbf{X}\right)^{-1} \mathbf{X}^{\top} \boldsymbol{\varepsilon}^{*}=\hat{\boldsymbol{\beta}}_{\mathrm{Z}}-\boldsymbol{\beta}_{0}
$$

where $\boldsymbol{\varepsilon}^{*}=\mathbf{Y}^{*}-\mathbf{X} \boldsymbol{\beta}_{0}, \hat{\boldsymbol{\beta}}_{\mathrm{Z}}=\left(\mathbf{X}^{\top} \mathbf{X}\right)^{-1} \mathbf{X}^{\top} \mathbf{Y}^{*}, \mathbf{D}_{1}(\boldsymbol{\alpha})=\operatorname{diag}\left(\alpha_{1}^{-2}, \ldots, \alpha_{q}^{-2}\right)$ and $\mathbf{D}_{2}(\boldsymbol{\gamma})=\operatorname{diag}\left(\gamma_{1}^{-2}, \ldots, \gamma_{p_{n}-q}^{-2}\right)$.

Lemma 1 Let $\delta_{n}$ be a sequence of positive real numbers satisfying $\delta_{n} \rightarrow \infty$ and $p_{n} \delta_{n}^{2} / \lambda_{n} \rightarrow 0$. Define $\mathbf{H}_{n}=\{\boldsymbol{\beta} \in$ $\left.\mathbb{R}^{p_{n}}:\left\|\boldsymbol{\beta}-\boldsymbol{\beta}_{0}\right\| \leq \delta_{n} \sqrt{p_{n} / n}\right\}$ and $\mathbf{H}_{n 1}=\left\{\boldsymbol{\alpha} \in \mathbb{R}^{q}:\left\|\boldsymbol{\alpha}-\boldsymbol{\beta}_{01}\right\| \leq \delta_{n} \sqrt{p_{n} / n}\right\}$. Assume conditions (C1)-(C5) hold. Then, with probability tending to 1 , we have

(a) $\sup _{\boldsymbol{\beta} \in \mathbf{H}_{n}}\left\|\boldsymbol{\gamma}^{*}\right\| /\|\boldsymbol{\gamma}\|<1 / C_{0}$, for some constant $C_{0}>1$;

(b) $g$ is a mapping from $\mathbf{H}_{n}$ to itself.

Proof We first prove part (a).

First, under $\lambda_{n} / \sqrt{n} \rightarrow 0$ and $p_{n} \delta_{n}^{2} / \lambda_{n} \rightarrow 0$, we have $\delta_{n} \sqrt{p_{n} / n} \rightarrow 0$.

Let $\hat{\boldsymbol{\beta}}_{\mathrm{Z}}=\left(\mathbf{X}^{\top} \mathbf{X}\right)^{-1} \mathbf{X}^{\top} \mathbf{Y}^{*}, \omega_{j i}=\left(\left(\mathbf{X}^{\top} \mathbf{X}\right)^{-1} \mathbf{X}^{\top}\right)_{j i}, \mu_{j}^{*}=\sum_{i} \omega_{j i} \int_{0}^{T_{n}} F_{i} d t$ and $\boldsymbol{\mu}=\left(\mu_{1}^{*}, \mu_{2}^{*}, \ldots, \mu_{p n}^{*}\right)$. For any $p_{n}$-vector $\mathbf{b}_{n}$ which $\left\|\mathbf{b}_{n}\right\| \leq 1$, define $\left.t_{n}^{2}=\mathbf{b}_{n}^{\top} \boldsymbol{\Omega}(\infty)\right) \mathbf{b}_{n}$. Then, we have $\sqrt{n} t_{n}^{-1} \mathbf{b}_{n}^{\top}\left(\hat{\boldsymbol{\beta}}_{\mathrm{Z}}-\boldsymbol{\mu}\right) \rightarrow_{D} N(0,1)$. This result can be proved using similar techniques to those used in the proof of Theorem 3.1 of Zhou (1992) along the same lines as outlined below: First, we separate $\mathbf{b}_{n}^{\top}\left(\hat{\boldsymbol{\beta}}_{\mathrm{Z}}-\boldsymbol{\mu}\right)$ like (3.6) in Zhou (1992) with a main term $S_{\boldsymbol{\beta}}\left(T^{n}\right)$ and a remainder term $S S_{\boldsymbol{\beta}}\left(T^{n}\right)$, i.e., $\mathbf{b}_{n}^{\top}\left(\hat{\boldsymbol{\beta}}_{\mathbf{Z}}-\boldsymbol{\mu}\right)=S_{\boldsymbol{\beta}}\left(T^{n}\right)+S S_{\boldsymbol{\beta}}\left(T^{n}\right)$, where $S_{\boldsymbol{\beta}}\left(T^{n}\right)$ is a weighted sum of $\hat{H}(t)-H(t)$ and $\hat{G}(t)-G(t)$; and $S S_{\boldsymbol{\beta}}\left(T^{n}\right)$ is a weighted sum of $(\hat{H}(t)-H(t))(\hat{G}(t)-G(t))$ and $(\hat{H}(t)-H(t))(\hat{H}(t)-H(t))$. Second, under conditions $(\mathrm{C} 2)$ and (C3), one can show that $\sqrt{n} S S_{\boldsymbol{\beta}}\left(T^{n}\right)$ is negligible. Finally, by applying the martingale central limit theorem and conditions $(\mathrm{C} 1)$ and $(\mathrm{C} 4)$, we establish the asymptotic normality of $\sqrt{n} S_{\boldsymbol{\beta}}\left(T^{n}\right)$. By condition $(\mathrm{C} 1)$ and $(\mathrm{C} 2)$, we have $\sqrt{n} t_{n}^{-1} \mathbf{b}_{n}^{\top}\left(\boldsymbol{\beta}_{0}-\boldsymbol{\mu}\right)=o_{p}(1)$, for $\mathbf{b}_{n}=\mathbf{e}_{i}=(0, \ldots, 1,0, \ldots, 0)$. Hence, we have $\left\|\hat{\boldsymbol{\beta}}_{\mathrm{Z}}-\boldsymbol{\beta}_{0}\right\|^{2}=O_{p}\left(p_{n} / n\right)$.

It then follows from (11) that

$$
\sup _{\beta \in \mathbf{H}_{n}}\left\|\boldsymbol{\gamma}^{*}+\lambda_{n} \mathbf{A}_{12}^{\top} \mathbf{D}_{1}(\boldsymbol{\alpha}) \boldsymbol{\alpha}^{*} / n+\lambda_{n} \mathbf{A}_{22} \mathbf{D}_{2}(\boldsymbol{\gamma}) \boldsymbol{\gamma}^{*} / n\right\|=O_{p}\left(\sqrt{p_{n} / n}\right)
$$

Note that $\left\|\boldsymbol{\alpha}-\boldsymbol{\beta}_{01}\right\| \leq \delta_{n}\left(p_{n} / n\right)^{1 / 2}$ and $\left\|\boldsymbol{\alpha}^{*}\right\| \leq\|g(\boldsymbol{\beta})\| \leq\left\|\hat{\boldsymbol{\beta}}_{\mathrm{Z}}\right\|=O_{p}\left(\sqrt{p_{n}}\right)$. By assumptions (C4) and (C5), we have

$$
\begin{aligned}
\sup _{\boldsymbol{\beta} \in \mathbf{H}_{n}}\left\|\lambda_{n} \mathbf{A}_{12}^{\top} \mathbf{D}_{1}(\boldsymbol{\alpha}) \boldsymbol{\alpha}^{*} / n\right\| & \leq \frac{\lambda_{n}}{n}\left\|\mathbf{A}_{12}^{\top}\right\| \sup _{\boldsymbol{\beta} \in \mathbf{H}_{n}}\left\|\mathbf{D}_{1}(\boldsymbol{\alpha}) \boldsymbol{\alpha}^{*}\right\| \\
& \leq \sqrt{2} \tilde{C} \frac{\lambda_{n}}{n} \frac{a_{1}}{a_{0}^{2}} \sup _{\boldsymbol{\beta} \in \mathbf{H}_{n}}\left\|\boldsymbol{\alpha}^{*}\right\|=o_{p}\left(\sqrt{p_{n} / n}\right),
\end{aligned}
$$

where the second inequality uses the fact $\left\|\mathbf{A}_{12}^{\top}\right\| \leq \sqrt{2} \tilde{C}$, which follows from the inequality $\left\|\mathbf{A}_{12} \mathbf{A}_{12}^{\top}\right\|-\left\|\mathbf{A}_{11}^{2}\right\| \leq$ $\left\|\mathbf{A}_{11}^{2}+\mathbf{A}_{12} \mathbf{A}_{21}\right\| \leq\left\|\boldsymbol{\Sigma}_{n}^{-2}\right\|<\tilde{C}^{2}$. Combining (12) and (13) gives

$$
\sup _{\boldsymbol{\beta} \in \mathbf{H}_{n}}\left\|\boldsymbol{\gamma}^{*}+\lambda_{n} \mathbf{A}_{22} \mathbf{D}_{2}(\boldsymbol{\gamma}) \boldsymbol{\gamma}^{*} / n\right\|=O_{p}\left(\sqrt{p_{n} / n}\right)
$$


Note that $\mathbf{A}_{22}=\sum_{i=1}^{p_{n}-q} \tau_{2 i} \mathbf{u}_{2 i} \mathbf{u}_{2 i}^{\top}$ is positive definite and by the singular value decomposition, , where $\tau_{2 i}$ and $\mathbf{u}_{2 i}$ are eigenvalues and eigenvectors of $\mathbf{A}_{22}$. Then, since $1 / \tilde{C}<\tau_{2 i}<\tilde{C}$, we have

$$
\begin{aligned}
\frac{\lambda_{n}}{n}\left\|\mathbf{A}_{22} \mathbf{D}_{2}(\boldsymbol{\gamma}) \boldsymbol{\gamma}^{*}\right\| & =\frac{\lambda_{n}}{n}\left\|\sum_{i=1}^{p_{n}-q} \tau_{2 i} \mathbf{u}_{2 i} \mathbf{u}_{2 i}^{\top} \mathbf{D}_{2}(\boldsymbol{\gamma}) \boldsymbol{\gamma}^{*}\right\|=\frac{\lambda_{n}}{n}\left\{\sum_{i=1}^{p_{n}-q} \tau_{2 i}^{2}\left\|\mathbf{u}_{2 i}^{\top} \mathbf{D}_{2}(\boldsymbol{\gamma}) \boldsymbol{\gamma}^{*}\right\|^{2}\right\}^{1 / 2} \\
& \geq \frac{\lambda_{n}}{n} \frac{1}{\tilde{C}}\left\{\sum_{i=1}^{p_{n}-q}\left\|\mathbf{u}_{2 i}^{\top} \mathbf{D}_{2}(\boldsymbol{\gamma}) \boldsymbol{\gamma}^{*}\right\|^{2}\right\}^{1 / 2}=\frac{1}{\tilde{C}}\left\|\lambda_{n} \mathbf{D}_{2}(\boldsymbol{\gamma}) \boldsymbol{\gamma}^{*} / n\right\|
\end{aligned}
$$

This, together with (14) and (C4), implies that with probability tending to 1 ,

$$
\frac{1}{\tilde{C}}\left\|\lambda_{n} \mathbf{D}_{2}(\boldsymbol{\gamma}) \boldsymbol{\gamma}^{*} / n\right\|-\left\|\boldsymbol{\gamma}^{*}\right\| \leq \delta_{n} \sqrt{p_{n} / n}
$$

Let $\mathbf{d}_{\gamma * / \gamma}=\left(\gamma_{1}^{*} / \gamma_{1}, \ldots, \gamma_{p_{n}-q}^{*} / \gamma_{p_{n}-q}\right)^{\top}$. Because $\|\gamma\| \leq \delta_{n} \sqrt{p_{n} / n}$, we have

$$
\frac{1}{\tilde{C}}\left\|\frac{\lambda_{n}}{n} \mathbf{D}_{2}(\boldsymbol{\gamma}) \boldsymbol{\gamma}^{*}\right\|=\frac{1}{\tilde{C}} \frac{\lambda_{n}}{n}\left\|\left\{\mathbf{D}_{2}(\boldsymbol{\gamma})\right\}^{1 / 2} \mathbf{d}_{\boldsymbol{\gamma} * / \boldsymbol{\gamma}}\right\| \geq \frac{1}{\tilde{C}} \frac{\lambda_{n}}{n} \frac{\sqrt{n}}{\delta_{n} \sqrt{p_{n}}}\left\|\mathbf{d}_{\boldsymbol{\gamma} * / \boldsymbol{\gamma}}\right\|
$$

and

$$
\left\|\boldsymbol{\gamma}^{*}\right\|=\left\|\mathbf{D}_{2}(\boldsymbol{\gamma})^{-1 / 2} \mathbf{d}_{\boldsymbol{\gamma} * / \boldsymbol{\gamma}}\right\| \leq \frac{\delta_{n} \sqrt{p_{n}}}{\sqrt{n}}\left\|\mathbf{d}_{\boldsymbol{\gamma} * / \boldsymbol{\gamma}}\right\| .
$$

Combining (15), (16) and (17), we have that with probability tending to 1 ,

$$
\left\|\mathbf{d}_{\boldsymbol{\gamma} * / \boldsymbol{\gamma}}\right\| \leq \frac{1}{\lambda_{n} /\left(p_{n} \delta_{n}^{2} \tilde{C}\right)-1}<1 / C_{0}
$$

for some constant $C_{0}>1$ provided that $\lambda_{n} /\left(p_{n} \delta_{n}^{2}\right) \rightarrow \infty$.

It is worth noting that $\operatorname{Pr}\left(\left\|\mathbf{d}_{\boldsymbol{\gamma} * / \boldsymbol{\gamma}}\right\| \rightarrow 0\right) \rightarrow 1$, as $n \rightarrow \infty$. Furthermore, with probability tending to 1 ,

$$
\left\|\boldsymbol{\gamma}^{*}\right\| \leq\left\|\mathbf{d}_{\boldsymbol{\gamma}^{*} / \boldsymbol{\gamma}}\right\| \max _{1 \leq j \leq\left(p_{n}-q\right)}\left|\boldsymbol{\gamma}_{j}\right| \leq\left\|\mathbf{d}_{\boldsymbol{\gamma}^{*} / \boldsymbol{\gamma}}\right\| \times\|\boldsymbol{\gamma}\| \leq\|\boldsymbol{\gamma}\| / C_{0}
$$

This proves part (a).

Next we prove part (b). First, it is easy to see from (17) and (18) that, as $n \rightarrow \infty$,

$$
\operatorname{Pr}\left(\left\|\gamma^{*}\right\| \leq \delta_{n} \sqrt{p_{n} / n}\right) \rightarrow 1
$$

Then, by (11), we have

$$
\sup _{\boldsymbol{\beta} \in \mathbf{H}_{n}}\left\|\boldsymbol{\alpha}^{*}-\boldsymbol{\beta}_{01}+\lambda_{n} \mathbf{A}_{11} \mathbf{D}_{1}(\boldsymbol{\alpha}) \boldsymbol{\alpha}^{*} / n+\lambda_{n} \mathbf{A}_{12} \mathbf{D}_{2}(\boldsymbol{\gamma}) \boldsymbol{\gamma}^{*} / n\right\|=O_{p}\left(\sqrt{p_{n} / n}\right) .
$$

Similar to (13), it is easily to verify that

$$
\sup _{\boldsymbol{\beta} \in \mathbf{H}_{n}}\left\|\lambda_{n} \mathbf{A}_{11} \mathbf{D}_{1}(\boldsymbol{\alpha}) \boldsymbol{\alpha}^{*} / n\right\|=o_{p}\left(\sqrt{p_{n} / n}\right) .
$$

Moreover, with probability tending to 1 ,

$$
\sup _{\boldsymbol{\beta} \in \mathbf{H}_{n}}\left\|\lambda_{n} \mathbf{A}_{12} \mathbf{D}_{2}(\boldsymbol{\gamma}) \boldsymbol{\gamma}^{*} / n\right\| \leq \frac{\lambda_{n}}{n} \sup _{\boldsymbol{\beta} \in \mathbf{H}_{n}}\left\|\mathbf{D}_{2}(\boldsymbol{\gamma}) \boldsymbol{\gamma}^{*}\right\| \times\left\|\mathbf{A}_{12}\right\| \leq 2 \sqrt{2} \tilde{C}^{2} \delta_{n} \sqrt{p_{n} / n},
$$

where the last step follows from (15), (19), and the fact that $\left\|\mathbf{A}_{12}\right\| \leq \sqrt{2} \tilde{C}$. It follows from (20), (21) and (22) that with probability tending to 1 ,

$$
\sup _{\boldsymbol{\beta} \in \mathbf{H}_{n}}\left\|\boldsymbol{\alpha}^{*}-\boldsymbol{\beta}_{01}\right\| \leq\left(2 \sqrt{2} \tilde{C}^{2}+1\right) \delta_{n} n^{-1 / 2} \sqrt{p_{n}} .
$$

Because $\delta_{n} \sqrt{p_{n}} / \sqrt{n} \rightarrow 0$, we have, as $n \rightarrow \infty$,

$$
\operatorname{Pr}\left(\boldsymbol{\alpha}^{*} \in \mathbf{H}_{n 1}\right) \rightarrow 1 .
$$

Combining (19) and (24) completes the proof of part (b). 
Lemma 2 Assume that (C1)-(C5) hold. For any q-vector $\mathbf{c}$ satisfying $\|\mathbf{c}\| \leq 1$, define $z^{2}=\mathbf{c}^{\top} \boldsymbol{\Omega}_{1} \mathbf{c}$ as in Theorem 1. Define

$$
f(\boldsymbol{\alpha})=\left\{\mathbf{X}_{1}^{\top} \mathbf{X}_{1}+\lambda_{n} \mathbf{D}_{1}(\boldsymbol{\alpha})\right\}^{-1} \mathbf{X}_{1}^{\top} \mathbf{Y}^{*}
$$

Then, with probability tending to 1 ,

(a) $f(\boldsymbol{\alpha})$ is a contraction mapping from $\mathbf{B}_{n} \equiv\left\{\boldsymbol{\alpha} \in \mathbb{R}^{q}:\left\|\boldsymbol{\alpha}-\boldsymbol{\beta}_{01}\right\| \leq \delta_{n} \sqrt{p_{n} / n}\right\}$ to itself;

(b) $\sqrt{n} z^{-1} \mathbf{c}^{\top}\left(\hat{\boldsymbol{\alpha}}^{\circ}-\boldsymbol{\beta}_{01}\right) \rightsquigarrow \mathcal{N}(0,1)$, where $\hat{\boldsymbol{\alpha}}^{\circ}$ is the unique fixed point of $f(\boldsymbol{\alpha})$ defined by

$$
\hat{\boldsymbol{\alpha}}^{\circ}=\left\{\mathbf{X}_{1}^{\top} \mathbf{X}_{1}+\lambda_{n} \mathbf{D}_{1}\left(\hat{\boldsymbol{\alpha}}^{\circ}\right)\right\}^{-1} \mathbf{X}_{1}^{\top} \mathbf{Y}^{*}
$$

Proof We first prove part (a). Note that (25) can be rewritten as

$$
f(\boldsymbol{\alpha})-\boldsymbol{\beta}_{01}+\frac{\lambda_{n}}{n} \boldsymbol{\Sigma}_{n 1}^{-1} \mathbf{D}_{1}(\boldsymbol{\alpha}) f(\boldsymbol{\alpha})=\hat{\boldsymbol{\beta}}_{1 Z}-\boldsymbol{\beta}_{01} .
$$

where $\hat{\boldsymbol{\beta}}_{1 \mathrm{Z}}=\left(\mathbf{X}_{1}^{\top} \mathbf{X}_{1}\right)^{-1} \mathbf{X}_{1}^{\top} \mathbf{Y}^{*}$. Then,

$$
\begin{gathered}
\sup _{\boldsymbol{\alpha} \in \mathbf{B}_{n}}\left\|f(\boldsymbol{\alpha})-\boldsymbol{\beta}_{01}+\left(\lambda_{n} / n\right) \boldsymbol{\Sigma}_{n 1}^{-1} \mathbf{D}_{1}(\boldsymbol{\alpha}) f(\boldsymbol{\alpha})\right\|=O_{p}(1 / \sqrt{n}) . \\
\sup _{\boldsymbol{\alpha} \in \mathbf{B}_{n}}\left\|\left(\lambda_{n} / n\right) \boldsymbol{\Sigma}_{n 1}^{-1} \mathbf{D}_{1}(\boldsymbol{\alpha}) f(\boldsymbol{\alpha})\right\|=o_{p}(1 / \sqrt{n}) .
\end{gathered}
$$

It follows from (26) and (27) that

$$
\sup _{\boldsymbol{\alpha} \in \mathbf{B}_{n}}\left\|f(\boldsymbol{\alpha})-\boldsymbol{\beta}_{01}\right\| \leq \delta_{n} / \sqrt{n},
$$

where $\delta_{n} \rightarrow \infty$ and $\delta_{n} / \sqrt{n} \rightarrow 0$. Then we can get

$$
\operatorname{Pr}\left(f(\boldsymbol{\alpha}) \in \mathbf{B}_{n}\right) \rightarrow 1, \text { as } n \rightarrow \infty .
$$

This means that $f$ is a mapping from the region $\mathbf{B}_{n}$ to itself.

Rewrite (25) as $\left\{\mathbf{X}_{1}^{\top} \mathbf{X}_{1}+\lambda_{n} \mathbf{D}_{1}(\boldsymbol{\alpha})\right\} f(\boldsymbol{\alpha})=\mathbf{X}_{1}^{\top} \mathbf{Y}^{*}$, then, we have

$$
\left(\boldsymbol{\Sigma}_{n 1}+\left(\lambda_{n} / n\right) \mathbf{D}_{1}(\boldsymbol{\alpha})\right) \dot{f}(\boldsymbol{\alpha})+\left(\lambda_{n} / n\right) \operatorname{diag}\left\{-2 f_{j}(\boldsymbol{\alpha}) / \alpha_{j}^{3}\right\}=0
$$

where $\dot{f}(\boldsymbol{\alpha})=\partial f(\boldsymbol{\alpha}) / \partial \boldsymbol{\alpha}^{\top}$ and $\operatorname{diag}\left\{\frac{-2 f_{j}(\boldsymbol{\alpha})}{\alpha_{j}^{3}}\right\}=\operatorname{diag}\left\{\frac{-2 f_{1}(\boldsymbol{\alpha})}{\alpha_{1}^{3}}, \ldots, \frac{-2 f_{q}(\boldsymbol{\alpha})}{\alpha_{q}^{3}}\right\}$. With the assumption $\lambda_{n} / \sqrt{n} \rightarrow 0$,

$$
\sup _{\boldsymbol{\alpha} \in \mathbf{B}_{n}}\left\|\left\{\boldsymbol{\Sigma}_{n 1}+\frac{\lambda_{n}}{n} \mathbf{D}_{1}(\boldsymbol{\alpha})\right\} \dot{f}(\boldsymbol{\alpha})\right\|=\sup _{\boldsymbol{\alpha} \in \mathbf{B}_{n}} \frac{2 \lambda_{n}}{n}\left\|\operatorname{diag}\left\{\frac{f_{j}(\boldsymbol{\alpha})}{\alpha_{j}^{3}}\right\}\right\|=o_{p}(1) \text {. }
$$

Write $\boldsymbol{\Sigma}_{n 1}=\sum_{i=1}^{q} \tau_{1 i} \mathbf{u}_{1 i} \mathbf{u}_{1 i}^{\top}$, where $\tau_{1 i}$ and $\mathbf{u}_{1 i}$ are eigenvalues and eigenvectors of $\boldsymbol{\Sigma}_{n 1}$. Then, by $(\mathrm{C} 4), 1 / \tilde{C}<$ $\tau_{1 i}<\tilde{C}$ for all $i$ and

$$
\begin{aligned}
\left\|\boldsymbol{\Sigma}_{n 1} \dot{f}(\boldsymbol{\alpha})\right\| & =\sup _{\|\mathbf{x}\|=1, \mathbf{x} \in R^{q}}\left\|\boldsymbol{\Sigma}_{n 1} \dot{f}(\boldsymbol{\alpha}) \mathbf{x}\right\|=\sup _{\|\mathbf{x}\|=1, \mathbf{x} \in R^{q}}\left\|\sum_{i=1}^{q} \lambda_{1 i} \mathbf{u}_{1 i} \mathbf{u}_{1 i}^{\top} \dot{f}(\boldsymbol{\alpha}) \mathbf{x}\right\| \\
& =\sup _{\|\mathbf{x}\|=1, \mathbf{x} \in R^{q}}\left(\sum_{i=1}^{q} \lambda_{1 i}^{2}\left\|\mathbf{u}_{1 i}^{\top} \dot{f}(\boldsymbol{\alpha}) \mathbf{x}\right\|^{2}\right)^{1 / 2} \geq \sup _{\|\mathbf{x}\|=1, \mathbf{x} \in R^{q}} \frac{1}{\tilde{C}}\left(\sum_{i=1}^{q}\left\|\mathbf{u}_{1 i}^{\top} \dot{f}(\boldsymbol{\alpha}) \mathbf{x}\right\|^{2}\right)^{1 / 2} \\
& =\sup _{\|\mathbf{x}\|=1, \mathbf{x} \in R^{q}} \frac{1}{\tilde{C}}\|\dot{f}(\boldsymbol{\alpha}) \mathbf{x}\|=\frac{1}{\tilde{C}}\|\dot{f}(\boldsymbol{\alpha})\| .
\end{aligned}
$$

Therefore, it follows from $\boldsymbol{\alpha} \in \mathbf{B}_{n}$, (32) and (C4) that

$$
\begin{aligned}
\left\|\left\{\boldsymbol{\Sigma}_{n 1}+\left(\lambda_{n} / n\right) \mathbf{D}_{1}(\boldsymbol{\alpha})\right\} \dot{f}(\boldsymbol{\alpha})\right\| & \geq\left\|\boldsymbol{\Sigma}_{n 1} \dot{f}(\boldsymbol{\alpha})\right\|-\left\|\left(\lambda_{n} / n\right) \mathbf{D}_{1}(\boldsymbol{\alpha}) \dot{f}(\boldsymbol{\alpha})\right\| \\
& \geq(1 / \tilde{C})\|\dot{f}(\boldsymbol{\alpha})\|-\left(\lambda_{n} / n\right) \cdot a_{0}^{-2}\|\dot{f}(\boldsymbol{\alpha})\|,
\end{aligned}
$$


This, together with (31) and the fact $\lambda_{n} / n \rightarrow 0$, implies that

$$
\sup _{\boldsymbol{\alpha} \in \mathbf{B}_{n}}\|\dot{f}(\boldsymbol{\alpha})\|=o_{p}(1)
$$

Finally, we can get the conclusion in part (a) from (29) and (33).

Next we prove part (b). Write

$$
\begin{aligned}
n^{1 / 2} z^{-1} \mathbf{c}^{\top}\left(\hat{\boldsymbol{\alpha}}^{\circ}-\boldsymbol{\beta}_{01}\right) & =n^{1 / 2} z^{-1} \mathbf{c}^{\top}\left[\left\{\boldsymbol{\Sigma}_{n 1}+\frac{\lambda_{n}}{n} \mathbf{D}_{1}\left(\hat{\boldsymbol{\alpha}}^{\circ}\right)\right\}^{-1} \boldsymbol{\Sigma}_{n 1}-\mathbf{I}_{q_{n}}\right] \boldsymbol{\beta}_{01} \\
& +n^{-1 / 2} z^{-1} \mathbf{c}^{\top}\left\{\boldsymbol{\Sigma}_{n 1}+\frac{\lambda_{n}}{n} \mathbf{D}_{1}\left(\hat{\boldsymbol{\alpha}}^{\circ}\right)\right\}^{-1} \mathbf{X}_{1}^{\top} \boldsymbol{\varepsilon}^{*} \equiv I_{1}+I_{2} .
\end{aligned}
$$

By the first order resolvent expansion formula

$$
(\mathbf{H}+\boldsymbol{\Delta})^{-1}=\mathbf{H}^{-1}-\mathbf{H}^{-1} \boldsymbol{\Delta}(\mathbf{H}+\boldsymbol{\Delta})^{-1},
$$

the first term on the right hand side of equation (34) can be rewritten as

$$
I_{1}=-z^{-1} \mathbf{c}^{\top} \boldsymbol{\Sigma}_{n 1}^{-1} \frac{\lambda_{n}}{\sqrt{n}} \mathbf{D}_{1}\left(\hat{\boldsymbol{\alpha}}^{\circ}\right)\left\{\boldsymbol{\Sigma}_{n 1}+\frac{\lambda_{n}}{n} \mathbf{D}_{1}\left(\hat{\boldsymbol{\alpha}}^{\circ}\right)\right\}^{-1} \boldsymbol{\Sigma}_{n 1} \boldsymbol{\beta}_{01} .
$$

Hence, by the assumption (C4) and (C5), we have

$$
\left\|I_{1}\right\| \leq \frac{\lambda_{n}}{\sqrt{n}} z^{-1} a_{0}^{-2}\left\|\boldsymbol{\Sigma}_{n 1}^{-1} \boldsymbol{\beta}_{01}\right\|=O_{p}\left(\lambda_{n} / \sqrt{n}\right) \rightarrow 0 .
$$

Furthermore, applying the first order resolvent expansion formula, it can be shown that

$$
\begin{aligned}
I_{2} & =\frac{z^{-1}}{\sqrt{n}} \mathbf{c}^{\mathrm{T}} \boldsymbol{\Sigma}_{n 1}^{-1} \mathbf{X}_{1}^{\mathrm{T}} \varepsilon^{*}+o_{p}(1) \\
& =\frac{z^{-1}}{\sqrt{n}} \mathbf{c}^{\mathrm{T}} \boldsymbol{\Sigma}_{n 1}^{-1} \mathbf{X}_{1}^{\mathrm{T}}\left(\mathbf{Y}^{*}-\mathbf{X}_{1} \boldsymbol{\mu}+\mathbf{X}_{1} \boldsymbol{\mu}-\mathbf{X}_{1} \boldsymbol{\beta}_{01}\right)+o_{p}(1) \\
& =\sqrt{n} z^{-1} \mathbf{c}^{\mathrm{T}}\left(\hat{\boldsymbol{\beta}}_{1 \mathrm{Z}}-\boldsymbol{\mu}_{1}+\boldsymbol{\mu}_{1}-\boldsymbol{\beta}_{01}\right)+o_{p}(1)
\end{aligned}
$$

where $\boldsymbol{\mu}_{1}=\left(\mu_{1}^{*}, \mu_{2}^{*}, \ldots, \mu_{q}^{*}\right) . I_{2}$ converges in distribution to $N(0,1)$ by the Lindeberg-Feller central limit theorem. Finally, combining (34), (35), and (36) proves part (b).

Proof of Theorem 1. Given the initial ridge estimator $\hat{\boldsymbol{\beta}}^{(0)}$ in (4), we have

$$
\begin{aligned}
\hat{\boldsymbol{\beta}}^{(0)}-\boldsymbol{\beta}_{0} & =\left[\left(\boldsymbol{\Sigma}_{n}+\frac{\xi_{n}}{n} \mathbf{I}_{p_{n}}\right)^{-1} \boldsymbol{\Sigma}_{n}-\mathbf{I}_{p_{n}}\right] \boldsymbol{\beta}_{0}+\left(\boldsymbol{\Sigma}_{n}+\frac{\xi_{n}}{n} \mathbf{I}_{p_{n}}\right)^{-1} \mathbf{X}^{\top} \boldsymbol{\varepsilon}^{*} / n . \\
& \equiv \mathbf{T}_{1}+\mathbf{T}_{2} .
\end{aligned}
$$

By the first order resolvent expansion formula and $\xi_{n} / \sqrt{n} \rightarrow 0$,

$$
\left\|\mathbf{T}_{1}\right\|=\left\|-\boldsymbol{\Sigma}_{n}^{-1} \frac{\xi_{n}}{n}\left(\boldsymbol{\Sigma}_{n}+\frac{\xi_{n}}{n} \mathbf{I}_{p_{n}}\right)^{-1} \boldsymbol{\Sigma}_{n} \boldsymbol{\beta}_{0}\right\| \leq \tilde{C}^{3} \frac{\xi_{n} a_{1} \sqrt{p_{n}}}{n}=o_{p}\left(\sqrt{\frac{p_{n}}{n}}\right) .
$$

It is easy to see that $\left\|\mathbf{T}_{2}\right\|=O_{p}\left(\sqrt{p_{n} / n}\right)$. Thus $\left\|\hat{\boldsymbol{\beta}}^{(0)}-\boldsymbol{\beta}_{0}\right\|=O_{p}\left(\left(p_{n} / n\right)^{1 / 2}\right)$. This, combined with part (a) of Lemma 1, implies that

$$
\operatorname{Pr}\left(\lim _{k \rightarrow \infty} \hat{\gamma}^{(k)}=0\right) \rightarrow 1 \text {. }
$$

Hence, to prove part (i) of Theorem 1, it is sufficient to show that

$$
\operatorname{Pr}\left(\lim _{k \rightarrow \infty}\left\|\hat{\boldsymbol{\alpha}}^{(k)}-\hat{\boldsymbol{\alpha}}^{\circ}\right\|=0\right) \rightarrow 1,
$$


where $\hat{\boldsymbol{\alpha}}^{\circ}$ is the fixed point of $f(\boldsymbol{\alpha})$ defined in part (b) of Lemma 2.

Define $\boldsymbol{\gamma}^{*}=0$ if $\boldsymbol{\gamma}=0$, for any $\boldsymbol{\alpha} \in \mathbf{B}_{n}$,

$$
\lim _{\boldsymbol{\gamma} \rightarrow 0} \boldsymbol{\gamma}^{*}(\boldsymbol{\alpha}, \boldsymbol{\gamma})=0
$$

Combining (41) with the fact

$$
\left(\begin{array}{cc}
\mathbf{X}_{1}^{\top} \mathbf{X}_{1}+\lambda_{n} \mathbf{D}_{1}(\boldsymbol{\alpha}) & \mathbf{X}_{1}^{\top} \mathbf{X}_{2} \\
\mathbf{X}_{2}^{\top} \mathbf{X}_{1} & \mathbf{X}_{2}^{\top} \mathbf{X}_{2}+\lambda_{n} \mathbf{D}_{2}(\boldsymbol{\gamma})
\end{array}\right)\left(\begin{array}{l}
\boldsymbol{\alpha}^{*} \\
\boldsymbol{\gamma}^{*}
\end{array}\right)=\left(\begin{array}{l}
\mathbf{X}_{1}^{\top} \mathbf{Y}^{*} \\
\mathbf{X}_{2}^{\top} \mathbf{Y}^{*}
\end{array}\right)
$$

implies that for any $\boldsymbol{\alpha} \in \mathbf{B}_{n}$,

$$
\lim _{\boldsymbol{\gamma} \rightarrow 0} \boldsymbol{\alpha}^{*}(\boldsymbol{\alpha}, \boldsymbol{\gamma})=\left\{\mathbf{X}_{1}^{\mathrm{T}} \mathbf{X}_{1}+\lambda_{n} \mathbf{D}_{1}(\boldsymbol{\alpha})\right\}^{-1} \mathbf{X}_{1} \mathbf{Y}^{*}=f(\boldsymbol{\alpha})
$$

Therefore, $g(\cdot)$ is continuous and thus uniformly continuous on the compact set $\boldsymbol{\beta} \in \mathbf{H}_{n}$. This, together with (39) and (42), implies that as $k \rightarrow \infty$,

$$
\eta_{k} \equiv \sup _{\boldsymbol{\alpha} \in \mathbf{B}_{n}}\left\|f(\boldsymbol{\alpha})-\boldsymbol{\alpha}^{*}\left(\boldsymbol{\alpha}, \hat{\boldsymbol{\gamma}}^{(k)}\right)\right\| \longrightarrow 0
$$

with probability tending to 1 .

Note that

$$
\begin{aligned}
\left\|\hat{\boldsymbol{\alpha}}^{(k+1)}-\hat{\boldsymbol{\alpha}}^{\circ}\right\| & =\left\|\boldsymbol{\alpha}^{*}\left(\hat{\boldsymbol{\beta}}^{(k)}\right)-\hat{\boldsymbol{\alpha}}^{\circ}\right\| \leq\left\|\boldsymbol{\alpha}^{*}\left(\hat{\boldsymbol{\beta}}^{(k)}\right)-f\left(\hat{\boldsymbol{\alpha}}^{(k)}\right)\right\|+\left\|f\left(\hat{\boldsymbol{\alpha}}^{(k)}\right)-\hat{\boldsymbol{\alpha}}^{\circ}\right\| \\
& \leq \eta_{k}+\frac{1}{\tilde{C}}\left\|\hat{\boldsymbol{\alpha}}^{(k)}-\hat{\boldsymbol{\alpha}}^{\circ}\right\|,
\end{aligned}
$$

where the last step follows from $\left\|f\left(\hat{\boldsymbol{\alpha}}^{(k)}\right)-\hat{\boldsymbol{\alpha}}^{\circ}\right\|=\left\|f\left(\hat{\boldsymbol{\alpha}}^{(k)}\right)-f\left(\hat{\boldsymbol{\alpha}}^{\circ}\right)\right\| \leq(1 / \tilde{C})\left\|\hat{\boldsymbol{\alpha}}^{(k)}-\hat{\boldsymbol{\alpha}}^{\circ}\right\|$. Let $a_{k}=\left\|\hat{\boldsymbol{\alpha}}^{(k)}-\hat{\boldsymbol{\alpha}}^{\circ}\right\|$, for all $k \geq 0$. From (43), we can induce that with probability tending to 1 , for any $\epsilon>0$, there exists an positive integer $N$ such that for all $k>N,\left|\eta_{k}\right|<\epsilon$ and

$$
\begin{aligned}
a_{k+1} & \leq \frac{a_{k-1}}{\tilde{C}^{2}}+\frac{\eta_{k-1}}{\tilde{C}}+\eta_{k} \\
& \leq \frac{a_{1}}{\tilde{C}^{k}}+\frac{\eta_{1}}{\tilde{C}^{k-1}}+\cdots+\frac{\eta_{N}}{\tilde{C}^{k-N}}+\left(\frac{\eta_{N+1}}{\tilde{C}^{k-N-1}}+\cdots+\frac{\eta_{k-1}}{\tilde{C}}+\eta_{k}\right) \\
& \leq\left(a_{1}+\eta_{1}+\ldots+\eta_{N}\right) \frac{1}{\tilde{C}^{k-N}}+\frac{1-(1 / \tilde{C})^{k-N}}{1-1 / \tilde{C}} \epsilon \rightarrow 0, \text { as } k \rightarrow \infty .
\end{aligned}
$$

This proves (40).

Therefore, it immediately follows from (39) and (40) that the with probability tending to $1, \lim _{k \rightarrow \infty} \boldsymbol{\beta}^{(k)}=$ $\lim _{k \rightarrow \infty}\left(\hat{\boldsymbol{\alpha}}^{(k) \top}, \hat{\boldsymbol{\gamma}}^{(k)^{\top}}\right)^{\top}=\left(\hat{\boldsymbol{\alpha}}^{\mathrm{o \top}}, 0\right)^{\mathrm{T}}$, which completes the proof of part (i). This, in addition to part (b) of Lemma 2, proves part (ii) of Theorem 1. $\quad$

Proof of Theorem 2. Recall that $\hat{\boldsymbol{\beta}}^{*}=\lim _{k \rightarrow \infty} \hat{\boldsymbol{\beta}}^{(k+1)}$ and $\hat{\boldsymbol{\beta}}^{(k+1)}=\arg \min _{\boldsymbol{\beta}}\left\{Q\left(\boldsymbol{\beta} \mid \hat{\boldsymbol{\beta}}^{(k)}\right)\right\}$, where

$$
Q\left(\boldsymbol{\beta} \mid \hat{\boldsymbol{\beta}}^{(k)}\right)=\left\|\mathbf{Y}^{*}-\mathbf{X} \boldsymbol{\beta}\right\|^{2}+\lambda_{n} \sum_{\ell=1}^{p_{n}} \beta_{\ell}^{2} /\left\{\hat{\beta}_{\ell}^{(k)}\right\}^{2} .
$$

If $\beta_{\ell}^{*} \neq 0$ for $\ell \in\{i, j\}$, then $\hat{\boldsymbol{\beta}}^{*}$ must satisfy the following normal equations for $\ell \in\{i, j\}$ :

$$
-2 \mathbf{x}_{\ell}^{\top}\left\{\mathbf{Y}^{*}-\mathbf{X} \hat{\boldsymbol{\beta}}^{(k+1)}\right\}+2 \lambda_{n} \hat{\beta}_{\ell}^{(k+1)} /\left\{\hat{\beta}_{\ell}^{(k)}\right\}^{2}=0 .
$$

Thus, for $\ell \in\{i, j\}$,

$$
\hat{\beta}_{\ell}^{(k+1)} /\left\{\hat{\beta}_{\ell}^{(k)}\right\}^{2}=\mathbf{x}_{\ell}^{\top} \hat{\varepsilon}^{*(k+1)} / \lambda_{n},
$$

where $\hat{\boldsymbol{\varepsilon}}^{*(k+1)}=\mathbf{Y}^{*}-\mathbf{X} \hat{\boldsymbol{\beta}}^{(k+1)}$. Moreover, because

$$
\left\|\hat{\boldsymbol{\varepsilon}}^{*(k+1)}\right\|^{2}+\lambda_{n} \sum_{i=1}^{p_{n}} \frac{\hat{\beta}_{i}^{2}}{\tilde{\beta}_{i}^{2}}=Q\left(\hat{\boldsymbol{\beta}}^{(k+1)} \mid \hat{\boldsymbol{\beta}}^{(k)}\right) \leq Q\left(0 \mid \hat{\boldsymbol{\beta}}^{(k)}\right)=\left\|\mathbf{Y}^{*}\right\|^{2}
$$


we have

$$
\left\|\hat{\varepsilon}^{*(k+1)}\right\| \leq\left\|Y^{*}\right\|
$$

Letting $k \rightarrow \infty$ in (45) and (46), we have, for $\ell \in\{i, j\}$ and $\left\|\hat{\varepsilon}^{*}\right\| \leq\left\|\mathbf{Y}^{*}\right\|, \hat{\beta}_{\ell}^{*-1}=\mathbf{x}_{\ell}^{\top} \hat{\varepsilon}^{*} \lambda_{n}$, where $\hat{\varepsilon}^{*}=\mathbf{Y}^{*}-\mathbf{X} \hat{\boldsymbol{\beta}}^{*}$. Therefore,

$$
\left|\hat{\beta}_{i}^{*-1}-\hat{\beta}_{j}^{*-1}\right| \leq \frac{1}{\lambda_{n}}\left\|\mathbf{Y}^{*}\right\| \times\left\|\mathbf{x}_{i}-\mathbf{x}_{j}\right\|=\frac{1}{\lambda_{n}}\left\|\mathbf{Y}^{*}\right\| \sqrt{2\left(1-\rho_{i j}\right)} .
$$

